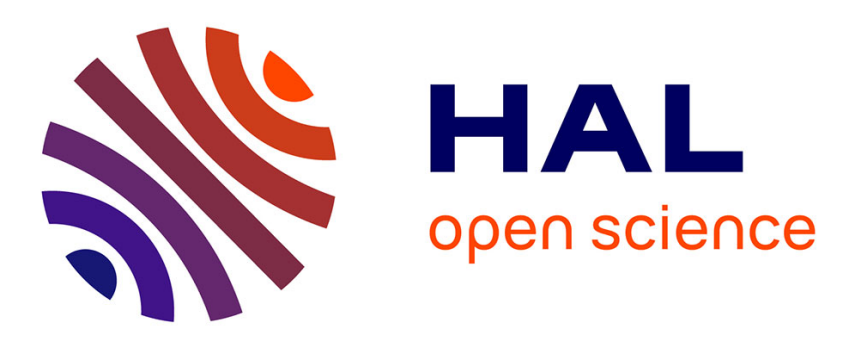

\title{
La chanson des mathématiques dans l'Edubba (Mésopotamie, début du deuxième millénaire avant notre ère) \\ Christine Proust
}

\section{- To cite this version:}

Christine Proust. La chanson des mathématiques dans l'Edubba (Mésopotamie, début du deuxième millénaire avant notre ère). Archives internationales d'histoire des sciences, 2017, Scientific Writings and Orality, 65/2, pp.19-49. hal-01464482

\section{HAL Id: hal-01464482 \\ https://hal.science/hal-01464482}

Submitted on 10 Feb 2017

HAL is a multi-disciplinary open access archive for the deposit and dissemination of scientific research documents, whether they are published or not. The documents may come from teaching and research institutions in France or abroad, or from public or private research centers.
L'archive ouverte pluridisciplinaire $\mathbf{H A L}$, est destinée au dépôt et à la diffusion de documents scientifiques de niveau recherche, publiés ou non, émanant des établissements d'enseignement et de recherche français ou étrangers, des laboratoires publics ou privés. 


\title{
La chanson des mathématiques dans l'Edubba
}

(Mésopotamie, début du deuxième millénaire avant notre ère)

\author{
Christine Proust ${ }^{1}$ \\ (Laboratoire SPHERE - CNRS \& Université Paris Diderot, Projet SAW)
}

\section{Introduction}

Les textes de Mésopotamie, notés en écriture cunéiforme sur des tablettes d'argile, comptent parmi les plus anciennes traces d'activités mathématiques qui soient parvenues jusqu'à nous. Il est difficile d'imaginer que des textes écrits il y a plus de quatre mille ans aient conservé, d'une façon qui nous soit intelligible, la trace de l'environnement oral dans lequel ils ont pu avoir été produits. Cependant, plusieurs raisons devraient nous inviter à un jugement moins pessimiste.

Une première raison réside dans la nature même des sources sur lesquels travaillent les historiens du Proche Orient Ancien, et parmi eux les historiens des mathématiques. Il s'agit de sources archéologiques, c'est-à-dire de documents écrits de la main même des acteurs dont on étudie les pratiques et les savoirs. L'étude des sources mésopotamiennes se présente ainsi de façon différente de celle des écrits mathématiques qui ont été transmis par une longue tradition savante, et dont on ne connait que des témoins tardifs, comme par exemple ceux de la Grèce ancienne. L'environnement oral éventuellement présent au moment de l'écriture de ces textes a-t-il pu laisser une empreinte ?

Une deuxième raison résulte du contexte dans lequel les textes qui sont parvenus jusqu'à nous ont été écrits. Il s'agit d'une période sensible de l'histoire intellectuelle de la Mésopotamie, où la tradition orale est confrontée à l'émergence de la pratique de l'écrit, qui s'impose non seulement comme moyen de communication à longue distance, mais aussi comme support privilégié de la transmission des connaissances. Cette pratique de l'écrit n'a pas pour autant réduit la place de la transmission orale. Piotr Michalowski souligne que l'enseignement oral est resté, à côté et parfois indépendamment de la pratique de l'écrit, une composante essentielle de la transmission des connaissances.

\footnotetext{
"It is also imperative to remind ourselves of the fact that oral schooling - formal and informal - can exist without writing or coexist with it, and that much knowledge and information can be transmitted from generation to generation in such contexts.” (Michalowski 2012: 39-40).
}

\footnotetext{
${ }^{1}$ Je remercie chaleureusement les relecteurs de cet article Micheline Duffaud, Piotr Michalowski, Luc Trouche, ainsi que les membres du projet SAW, qui m'ont permis de lui apporter de substantielles améliorations. Particulièrement précieuse fut l'assistance philologique de Piotr Michalowski, qui a m'a proposé sa propre traduction de certains passage de littérature sumérienne. Les éventuelles faiblesses ou erreurs sont de ma seule responsabilité. Les recherches nécessitées par ce chapitre ont été financées par l'European Research Council, dans le cadre du 7e programme de recherche de 1'Union Européenne (FP7/2007-2013), ERC Grant agreement n. 269804.
} 
Cette composante orale n'est cependant que très difficilement accessible à l'historien moderne, qui, dépendant des sources écrites, reste confronté à l'impossibilité de la restituer pleinement. Michalowski doute de la possibilité d'accéder à des processus cognitifs fins au travers de la documentation écrite disponible ${ }^{2}$. Par ailleurs, l'érudition ne se limitait pas aux savoirs fixés par écrit, mais incluait le théâtre, la liturgie ou la musique ${ }^{3}$. Bien que les phénomènes complexes d'oralité soient difficiles, voire impossibles, à percevoir au travers des sources écrites qui nous sont parvenues, il est cependant possible d'en détecter quelques formes particulières liées à la mémorisation.

Dans cette étude, dont le but est de rechercher des traces d'oralité dans les textes mathématiques, je me limiterai à l'examen des écrits qui ont été produits dans le contexte des écoles de scribes. Deux types de sources nous renseignent plus spécifiquement sur l'environnement oral: d'abord, il y a les textes littéraires qui étaient utilisés dans les écoles pour l'apprentissage de la langue sumérienne, et qui font allusion à diverses formes d'oralité ; ensuite il y a la production des jeunes apprentis scribes eux-mêmes. Exploiter le premier type de source soulève la question du statut de ces textes littéraires et de leur historicité. Dans quelle mesure des textes littéraires, écrits pour éduquer, voire pour édifier, reflètent-ils des pratiques réelles? A quelle époque ont-ils été composés, et quelle est la réalité qu'éventuellement ils pourraient refléter ? Peut-on en extraire des informations, ou bien ne doit-on pas les considérer comme de pures fictions, ou comme des idéalisations à but éducatif, ou encore comme l'écho d'un monde révolu ? Répondre à ces questions suppose une analyse du contexte scolaire, qui sera l'objet de la première partie de cette contribution $(\S 1)$.

Sur le deuxième type de sources, les tablettes scolaires, il s'agira d'analyser de possibles traces laissées dans les textes par les processus de mémorisation. Les archéologues qui ont fouillé les grands sites d'Irak, de Syrie et d'Iran, ont exhumé un très grand nombre de « tablettes scolaires », produites par de jeunes élèves scribes au cours des premières années de leur formation. L'analyse des traces de mémorisation laissées dans les textes scolaires mathématiques, principalement sous forme d'erreurs dans la restitution écrite des textes, sera l'objet de la deuxième partie de cette contribution $(\S 2)$.

\footnotetext{
2 "Moreover, many scholars now believe that these texts were learned by rote memorization and only committed to writing in exercise fashion, although a case can be made that in some learning environments direct copying from model tablets must have also been part of educational practice; this method of learning would become more prevalent in later periods" (Michalowski 2012: 40). Et un peu plus loin: "Such methods of instruction involve specific mental and physical processes, but the surviving documentation does not provide many clues concerning the cognitive aspects of such learning practices, nor does it give us access to the oral instruction, hermeneutic explanations and cultural commentary that must have accompanied the memorization and writing of specific exercises. Because of the limitations of the surviving documentation it is difficult to conceive of such learning in terms of "knowledge transfer" other than in strictly instrumental terms" (Michalowski 2012: 41).

${ }^{3}$ Veldhuis 1997 : 41, 131 ; Michalowski 2010. Par exemple, d'après Michalowski, l'enseignement de la musique ne relevait pas du curriculum des écoles de scribes où était enseignée l'écriture cunéiforme, mais d'une formation de type professionnel non basée sur l'écrit.
} 
La conclusion dégagera quelques hypothèses concernant le problème des écoles soulevé dans la première partie $(\S 1)$ à la lumière des indices sur les processus de mémorisation détectées dans les textes scolaires mathématiques $(\S 3)$.

\section{1- "J'ai appris l'art du scribe »: les écoles de scribes vues à travers les textes littéraires}

La majorité des textes mathématiques cunéiformes connus à ce jour a été produit dans le cadre des écoles de scribes qui se sont développées dans tout le Proche Orient Ancien à l'époque paléo-babylonienne (début $\mathrm{du} \quad 2^{\mathrm{e}}$ millénaire avant notre ère). Une quantité considérable de tablettes d'argile contenant des exercices d'écoliers nous documentent sur ces écoles. Le curriculum d'enseignement de certaines écoles a pu être reconstitué avec une grande précision ${ }^{4}$. Le mieux connu est celui des écoles de Nippur, la grande capitale culturelle de la Mésopotamie paléo-babylonienne, tout au moins le curriculum de celles parmi ces écoles qui étaient liées aux élites sociales et politiques. L'essentiel de la formation était consacré à l'apprentissage de l'écriture cunéiforme, de la langue sumérienne, et des mathématiques. Pour ce qui concerne l'apprentissage de l'écriture cunéiforme et de la langue sumérienne, ce curriculum consistait à apprendre, dans cet ordre, des syllabaires, et des listes de vocabulaire organisées sur un principe thématique, puis sur un principe graphique. Cet ensemble de listes constituait un programme cohérent que Niek Veldhuis (1997) dénomme le «niveau élémentaire ». Ensuite, un niveau intermédiaire consistait à assimiler des listes de proverbes et de modèles de contrats. Enfin, les textes littéraires sumériens, tout d'abord quatre textes simples (ou « Tétrade »), puis dix textes plus complexes (ou « Décade »), étaient étudiés dans un niveau avancé (voir Tinney 1999).

Le curriculum d'enseignement des mathématiques à Nippur a été reconstitué par Eleanor Robson $\left(2001\right.$; 2002) à partir du résultat des fouilles d'une maison particulière, ${ }^{5}$ et par la suite par moi-même sur la base de l'ensemble des tablettes mathématiques trouvées à Nippur (Proust 2007, 2008b). Le curriculum mathématique consistait, au niveau élémentaire, à assimiler, dans cet ordre, des listes et des tables métrologiques (énumérations de mesures de capacités, poids, surfaces et longueurs), et des tables numériques (inverses, multiplications, carrés, racines carrées et racines cubiques). Le niveau intermédiaire consistait à effectuer des

\footnotetext{
${ }^{4}$ Depuis les travaux pionniers de Benno Landsberger (1956), un grand nombre d'études ont été consacrées à la reconstitution du curriculum d'enseignement dans les écoles de scribes, notamment ceux d'Antoine Cavigneaux, qui décrit ainsi le Curriculum: «Suivant Landsberger, on peut reconstituer avec assez de certitude les premiers stades de l'enseignement dans $l^{\prime} E d u b b a \hat{~ d e ~ N i p p u r: ~ t u-t a-t i, ~} a-a$ («Silbenalphabet B»), Proto-Ea; pour le reste on ne peut guère que se baser sur les lignes d'appel; on peut supposer, sans certitude: Proto-Hh., Proto-Lü, Proto-Izi, Proto-Diri, 111-âzlag : aflaku, Ugu-mu et autres listes. Naturellement on étudiait plusieurs listes en même temps, ou bien une liste et d'autres matières, comme il ressort clairement du «type II» de Civil (MSL XII 27). » (Cavigneaux 1983). Ce sujet a connu un regain d'intérêt à la suite de la publication de la thèse de doctorat de Veldhuis (1997).

${ }^{5}$ Cette maison a été dénommée la « Maison F » par les archéologues qui l'ont exhumée. Cette maison est située dans le quartier des scribes de Nippur. On y a trouvé trouvés environ 1700 tablettes scolaires, essentiellement lexicales et littéraires; ces tablettes constituent le seul lot important de tablettes scolaires de Nippur dont le contexte archéologique est clair (Robson 2001).
} 
exercices de multiplication, d'inversion et de calcul de surface. Le niveau avancé en mathématiques est mal documenté, et portait probablement sur la résolution de listes de problèmes linéaires et quadratiques ${ }^{6}$. L'articulation des curricula littéraires et mathématiques à Nippur peut être très schématiquement représentée par le diagramme suivant (figure 1).

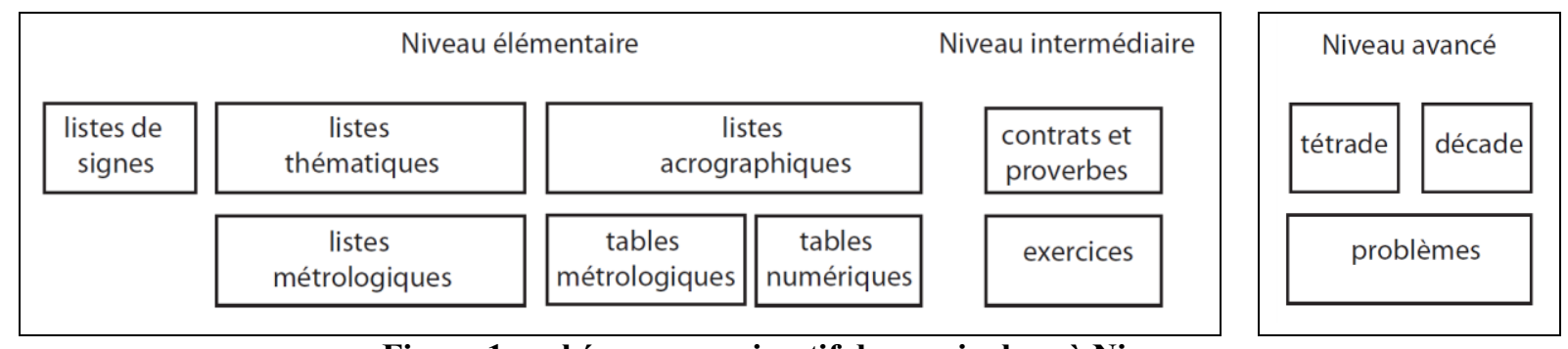

Figure 1 : schéma approximatif du curriculum à Nippur

Ce schéma trop bien rangé est très approximatif et présente une image simplifiée de pratiques beaucoup plus complexes et, sans doute, diversifiées selon les écoles. En particulier, les différents blocs représentés par des rectangles devraient en partie se chevaucher, voire se substituer l'un à l'autre ${ }^{7}$. De plus, les limites du «niveau intermédiaire » sont floues, et les curricula lexicaux et mathématiques ne coïncidaient pas nécessairement. Par exemple, on trouve des contrats et proverbes sur les mêmes tablettes que des tables métrologiques ou numériques $^{8}$. De plus, il faut insister sur le fait que tout le corpus mathématique connu n'entre pas dans cette structure curriculaire. En effet, la plupart des textes mathématiques érudits, bien que produits dans le contexte des écoles de scribes, ne sont pas des exercices d'écoliers ni des écrits d'étudiants avancés, ni même peut-être, pour certains, destinés à l'enseignement ${ }^{9}$.

Les sources disponibles offrent ainsi aux historiens modernes la possibilité d'une connaissance relativement précise, quoique limitée à certains aspects très particuliers, du contexte dans lequel ont été écrits et utilisés les textes lexicaux, mathématiques et littéraires à l'époque paléo-babylonienne. Pourtant, malgré l'abondance des sources, la nature des écoles de scribes paléo-babyloniennes reste une question controversée. Les écoles de scribes étaientelles des institutions, au sens d'entreprises à grande échelle, se déroulant dans des lieux spécifiquement dédiés à l'enseignement, régies par des règles standardisées et poursuivant des objectifs imposés par un pouvoir étatique, qu'il soit royal ou religieux ? Ou bien les écoles relevaient-elles d'initiatives privées locales, informelles, se déroulant essentiellement dans le cadre familial ? Une importante littérature a été consacrée à cette question ces dernières années ${ }^{10}$. En particulier, il a été mis en évidence une sorte de contradiction entre l'image

\footnotetext{
${ }^{6}$ Voir par exemple les catalogues et textes de procédure associés (YBC 4657, 4663 et 4662), publiés par Neugebauer et Sachs (1945: 66-75) et interprétés comme reflétant un curriculum dans Proust 2012. Ces tablettes ne proviennent cependant pas de Nippur, mais d'une autre cité de Mésopotamie méridionale.

${ }^{7}$ Il n'est pas exclus qu'un certain degré de spécialisation ait existé dans certaines écoles, et que, par exemple, certains scribes étudiaient les listes métrologiques alors que d'autres, plus orientés vers les mathématiques, étudiaient plutôt, à la place, les tables métrologiques. Notons aussi que dans la Maison F, les textes littéraires dominaient très largement les textes mathématiques, peu représentés.

${ }^{8}$ Pour une représentation plus précise du curriculum mathématique, voir Proust 2007 : 152, fig. 9.

${ }^{9}$ Voir, par exemple, la discussion sur la fonction de certaines listes d'énoncés de problèmes dans Proust 2014.

${ }^{10}$ Charpin 1986, 1989, 1990, Veldhuis 1997, Tanret 2002, George 2005, Robson 2008: §4.2.
} 
monumentale des écoles donnée par la littérature sumérienne, et les réalités matérielles fort modestes dont témoignent les restes archéologiques. Le débat est important pour notre propos car les textes littéraires produits dans les écoles fournissent la principale documentation disponible faisant explicitement référence à un environnement oral. La question se pose alors de savoir dans quelle mesure les textes littéraires offrent des témoignages à prendre en considération dans la présente enquête. Comme souligné dans l'introduction, une analyse de la nature des écoles et du statut des textes que ces écoles ont produits est un préalable nécessaire, et il est important de revenir sur les principaux arguments avancés dans les publications qui abordent ce « problème des écoles », pour reprendre l'expression de Dominique Charpin.

Par ailleurs, les historiens se sont souvent interrogés sur l'énigme que constitue la très faible quantité de sources scolaires datant de l'époque de la troisième dynastie d'Ur (dite Ur III, env. 2100-2000 av. notre ère), en comparaison de l'énorme documentation scolaire paléobabylonienne (env. 2000-1600 av. notre ère) ${ }^{11}$. Pourtant, de nombreux indices, y compris dans la documentation administrative, laissent penser que des écoles étaient très actives dès la période d'Ur III ${ }^{12}$. Les hymnes royaux écrits à la gloire des souverains de la troisième dynastie d'Ur attribuent à ces derniers la fondation des écoles de scribes, et vantent cette entreprise comme une de leurs réalisations les plus remarquables. Avoir fréquenté une Edubba, mot-àmot « maison des tablettes » en sumérien, est une marque de prestige, comme en témoigne par exemple de début de «L'hymne Šulgi B » ${ }^{13}$ :

11. Roi engendré de la semence d'un roi, né d'une reine, [je suis un....]

12. je suis Šulgi, noble enfant dont le destin a été fixé dès la matrice (de sa mère).

13. Lorsque j'étais un jeune garçon, alors que j'étais dans l'Edubba,

14. avec les tablettes de Sumer et d'Akkad, j'ai appris l'art du scribe.

15. Aucun des (autres) nobles enfants ne pouvaient écrire des tablettes comme moi,

16. parmi ceux qui fréquentaient le lieu de connaissance de l'art du scribe.

\footnotetext{
11 Voir notamment George 2005 et Friberg 2009.

12 George $2005: 6$.

13 Il n'existe pas d'édition critique récente des hymnes Šulgi B et Šulgi E (Cooper 2010). Les éditions disponibles sont celles de Castellino (1972) et du corpus en ligne d'ETCSL (http://etcsl.orinst.ox.ac.uk/section2/tr24202.htm). Je remercie Piotr Michalowski, qui m'a généreusement offert sa propre lecture du texte (communication personnelle, août 2014). Je donne ici une traduction en français que j'ai essayé de rendre la plus fidèle possible à la lecture de Piotr Michalowski (les maladresses éventuelles ce rendu en français sont de ma seule responsabilité), ainsi que la traduction originale de P. Michalowski :
}

A king — by royal seed engendered, born of a queen am I, I am Shulgi - a noble child whose future was already determined in (his divine mother's) fruitful womb! Once I was a young lad, when I was in the place of learning,

From the tablets of Sumer and Akkad I learned the scribal arts.

None among the (other) noble children could write tablets like me,

Among those who frequented the place of wisdom (to study) the scribal arts.

I fully mastered addition, subtraction counting and accounting:

Beautiful Nanibgal, that is Nidaba,

Swelled my wisdom and intelligence;

I am a learned scribe who forgets nothing. 
17. J'ai complètement achevé (l'apprentissage) de l'addition, la soustraction, les calculs et les comptes.

18. Belle Nanibgal, Nisaba

19. a étendu ma sagesse et mon intelligence;

20. je suis un scribe savant qui n'oublie rien.

Dans cet extrait, l'Edubba apparaît comme une école des princes (lignes 13-16), où les mathématiques et la comptabilité sont considérées comme des savoirs prestigieux (ligne 17). Il faut rappeler que c'est de la troisième dynastie d'Ur que datent les plus anciens textes mathématiques faisant un usage systématique de la notation sexagésimale positionnelle, et que ces textes semblent précisément émaner d'écoles de scribes. Les écoles d'Ur III auraient ainsi joué un rôle essentiel dans la constitution des premiers corpus mathématiques basés sur la notation positionnelle ${ }^{14}$. Comment expliquer alors que les écoles de scribes d'Ur III, si elles ont existé, n'aient laissé que très peu de traces hormis quelques passages lyriques dans les textes littéraires ? Bien sûr, une telle situation peut résulter du hasard des fouilles. Mais on peut envisager d'autres explications. La prise en compte de l'environnement d'oralité pourrait peutêtre d'offrir des hypothèses nouvelles.

\subsection{Des indices dans les textes Edubba?}

Parmi les documents qui nous renseignent sur les écoles de scribes, nous avons cité la littérature sumérienne, dont la majorité des sources connues provient des écoles de Nippur d'époque paléo-babylonienne. Cette littérature comprenait un genre de texte très en vogue dans ces écoles, les textes dits Edubba par les sumérologues. Ces compositions de tonalité humoristique décrivent des scènes de la vie quotidienne des écoliers. L'une de ces compositions, sans doute la plus connue, a été publiée pour la première fois en 1949 par Samuel Noah Kramer ${ }^{15}$, puis complétée et mise à jour au fil des découvertes de nouveaux fragments, et enfin intégrée à la base de données des textes littéraires sumériens sous le nom «Edubba 1 » (ETCSL 5.1.1). Une traduction en français a été publiée en ligne récemment par Pascal Attinger ${ }^{16}$. Ce récit a beaucoup influencé le regard porté sur les écoles de scribes paléobabyloniennes par les premiers historiens qui s'y sont intéressés. Le texte relate la journée d'un écolier, qui quitte le domicile familial le matin pour se rendre à l'école. Là, l'écolier rencontre tour à tour des professionnels spécialisés dans toutes sortes d'aspects de la vie de l'école : le directeur, le professeur de sumérien, divers responsables de la discipline, du maintien, de la cour, de la porte, etc., représentant pas moins de treize professions différentes. Le jeune écolier est pris en faute et frappé durement à chaque rencontre. Après que le malheureux a fait un compte rendu détaillé de ses déboires à son père, le soir venu, celui-ci convoque le directeur de l'école. A force de louanges et de somptueux cadeaux, le père obtient la bienveillance du directeur à l'égard de l'écolier récalcitrant. Au-delà de son aspect humoristique et des détails

\footnotetext{
${ }^{14}$ Les rares exemplaires connus de tablettes scolaires datables de façon quelque peu sûre d'Ur III sont sept tables d'inverses provenant de Nippur et de Tello, en Mésopotamie du sud (voir Ouyang et Proust à paraître et la bibliographie donnée dans cette publication).

15 Kramer 1949.

${ }^{16}$ Attinger 2009.
} 
pittoresques qu'il donne, ce texte laisse entrevoir l'image de l'école comme une institution de grande envergure, hors de la sphère domestique, dotée d'un personnel nombreux, et financée par les familles des écoliers. Mais quelle est l'historicité de telles anecdotes?

\subsection{Le problème des écoles}

Plusieurs historiens, notamment Dominique Charpin et, plus récemment, Andrew George $^{17}$ ont remis en question l'image des écoles donnée par un texte tel que celui que nous venons d'évoquer. Selon George, les textes dits Edubba, notamment les textes «Edubba 1 » (qui vient d'être évoqué) et «Dialogue 1 » (voir ci-dessous), ont ancré chez beaucoup d'historiens l'idée que l'école paléo-babylonienne, avec sa hiérarchie complexe, son personnel nombreux et son public étudiant important, était une sorte d'université laïque, une grande institution académique à caractère étatique. Cependant, selon lui, trois exemples contredisent la vision des écoles comme étant des institutions académiques : la Maison F de Nippur et les deux « écoles » d'Ur. ${ }^{18}$ A la suite de Charpin (1990), George souligne que la Maison F de Nippur et les maisons ayant abrité des écoles à Ur sont petites et privées, et que les textes scolaires ont souvent été trouvés mélangés à des textes administratifs. On n'a pas trouvé de grands édifices spécifiquement dédiés à l'enseignement à l'époque paléo-babylonienne. De ces éléments et d'autres indices, George en conclut qu'à Ur et Nippur ${ }^{19}$, l'enseignement était délivré à petite échelle, et les propriétaires de ces maisons enseignaient l'écriture chez eux à deux ou trois garçons, à savoir leurs propres fils et ceux de parents ou de collègues ${ }^{20}$. Selon Tanret (1982: 49), « l'idée d'un espace architectural réservé uniquement à l'enseignement, d'une salle de classe, nous paraît donc, pour cette période, un anachronisme ».

George propose une explication à cette apparente discordance entre les sources littéraires et archéologiques. Selon lui, les textes littéraires paléo-babyloniens témoignent d'une tradition plus ancienne. Pour George, contrairement à ce que pensaient Kramer et bien d'autres après lui, les textes utilisés dans les écoles paléo-babyloniennes ont été composés pendant la troisième dynastie d'Ur. George en conclut que les textes littéraires rapportent une image de l'école datant de cette période plus ancienne, au cours de laquelle se seraient développées de grandes institutions d'enseignement académique, notamment à Ur et à Nippur. L'Edubba sous la troisième dynastie d'Ur a dû être monumentale, aussi bien comme institution que dans son architecture, avec de grands édifices composés de nombreuses pièces, et, souligne George, il n'est pas difficile d'imaginer qu'une telle institution était soutenue par l'état. La conclusion de George est que l'Edubba était une institution typique de la période d'Ur III, et qu'elle avait disparu à l'époque paléo-babylonienne. George prédit même qu'on ne trouvera jamais de grands édifices dédiés à l'enseignement dans les niveaux archéologiques correspondant à l'époque paléo-babylonienne, mais qu'on en trouvera peut-être dans les couches plus anciennes,

\footnotetext{
${ }^{17}$ Charpin 1986, 1989, 1990, George 2005.

${ }^{18}$ Sur les deux écoles d'Ur, voir Charpin 1986.

${ }^{19}$ Des considérations similaires ont été faites concernant les écoles d'Isin (Wilcke 1987, p. 3), de Sippar (Gasche 1989, p. 19-20, 40-41), et de Tell Harmal (Miglus et Hussein, à paraître).

${ }^{20}$ Notons que les filles n'étaient pas exclues de l'enseignement (Lion et Robson 2006).
} 
correspondant à la période d'Ur III ou au tout début du deuxième millénaire (époque des dynasties d'Isin et de Larsa).

Eleanor Robson (2008 : 105), pour sa part, a fait très justement remarquer que les textes Edubba n'avaient certainement pas pour fonction d'informer sur les réalités des écoles de scribes, puisque ces réalités étaient précisément celles qui étaient vécues par leurs lecteurs ou utilisateurs. Les textes $E d u b b a$ ne sont pas des reportages, mais des écrits didactiques destinés à la fois à l'apprentissage du sumérien et au conditionnement idéologique des élites ${ }^{21}$. Cela signifie-t-il qu'il faut écarter les textes $E d u b b a$ en tant que source d'information sur les écoles paléo-babyloniennes ? Dans quelle mesure certains détails livrés par les textes Edubba sont-ils corroborés par d'autres types de sources? Quelles réalités recouvre le mot même d'institution dans le contexte de ces sociétés anciennes?

La tentative de caractérisation de l'école paléo-babylonienne pourrait s'avérer vaine car n'y avait probablement pas de modèle unique en Mésopotamie. Michalowski a attiré l'attention récemment sur la diversité des pratiques en matière d'enseignement ${ }^{22}$. Le cadre de l'enseignement était sans doute très différent à Nippur, qui revendiquait son indépendance par rapport au clergé, à Me Turan, dans la vallée de la Diyala en haute Mésopotamie, où la liturgie semble avoir fait partie de la formation des jeunes scribes (Cavigneaux 1999), ou à Kiš, en Mésopotamie centrale, où l'enseignement était plus utilitaire qu'à Nippur (Michalowski 2012 : 42). De plus, la discussion peut être biaisée par le sens même donné au mot institution, qui réunirait des attributs tels que le caractère monumental, un contrôle étatique et de grands effectifs. Or, l'effectif d'une école, le lieu où elle est installée, le contrôle du curriculum, sont des aspects différents et relativement indépendants, ou en tout cas à considérer séparément. Un enseignement à grande échelle ne signifie pas que des bâtiments monumentaux devaient l'accueillir. Une école peut fonctionner dans une maison privée tout en étant institutionnelle au sens où son personnel peut être professionnel, ses maîtres recevoir un salaire, son curriculum s'inscrire dans un projet étatique ou clérical. La question n'est donc pas de savoir si les écoles étaient des institutions, mais plutôt, par exemple, de savoir si les maîtres étaient des professionnels, quelles étaient leurs sources de revenu, quel était le nombre d'étudiants, quel

\footnotetext{
${ }^{21}$ Michalowski 1987, Veldhuis 1997 ; Michalowski 2012. L'article de 1987 de Michalowski développe une analyse très bourdieusienne des écoles de scribes, décrites comme un moule idéologique dont la finalité est la fabrication une élite sociale et politique. Ce point de vue a largement inspiré les recherches ultérieures sur les écoles de scribes, en particulier celles de Veldhuis (1997). Plus de vingt ans plus tard, Michalowski revient de façon critique sur son approche de 1987, qu'il assume tout en la nuançant. Dans sa nouvelle approche, Michalowski insiste sur l'idée que l'école n'est pas un tout monolithique, et il décrit d'importantes différenciations sociales et géographiques dans la formation des scribes. Il suggère de remplacer la notion de "stream of tradition", très populaire dans les études sur l'histoire intellectuelle de la Mésopotamie, par celle de « ebb and flow of multiple traditions » (Michalowski $2012: 40$ ).

${ }^{22}$ Michalowski distingue par exemple le scribe ordinaire (dub-sar, mot-à-mot celui qui écrit sur les tablettes d'argile) de l'érudit, qui, selon lui, est désigné par un autre terme (dumu-é-dub-ba-a, mot-à-mot, « scribe enfant de l'école », au sens de " scribe moulé par l'école »). Voir aussi une tentative pour mettre en évidence des sortes de « filières » dans les écoles de scribes dans Proust 2008a.
} 
était le degré de standardisation de l'enseignement. Il est probable qu'il n'y a pas de réponse uniforme à chacune de ces questions, mais autant de réponses différentes qu'il y a d'écoles.

Pour ce qui est de Nippur, l'énorme quantité de tablettes scolaires trouvées semble plaider pour une entreprise à grande échelle. Le fait que l'endroit où ont été trouvées les tablettes de la «Maison F » n'était pas un bâtiment monumental mais un groupe de petites maisons désordonnées n'apporte pas beaucoup d'éclaircissements quant à l'architecture, monumentale ou non, des écoles. En effet, on sait que l'enseignement de l'écriture avait lieu à l'extérieur des maisons, en particulier à cause des problèmes d'éclairage. Et on sait aussi que les tablettes scolaires n'étaient pas stockées ou recyclées sur le lieu exact où elles avaient été écrites. L'auteur du texte "Edubba 1 » a sans doute exagéré certains traits pour créer des effets comiques, comme par exemple l'intervention d'une multitude de professionnels chargés de fonctions improbables. Cependant, tous les détails ne sont pas nécessairement à ignorer en tant qu'indices. En particulier, il est tout à fait possible qu'à Nippur à l'époque paléo-babylonienne, les maîtres des écoles de scribes aient effectivement été des professionnels tirant leurs revenus des familles des écoliers, et que l'enseignement se soit déroulé dans un lieu dédié, séparé des lieux d'habitation, comme en témoigne le texte « Edubba $1 »$.

\subsection{Le curriculum décrit dans les textes Edubba}

Les textes Edubba reflètent-ils des réalités corroborées par d'autres types de sources? On peut répondre à cette question sur au moins un aspect : celui du curriculum d'enseignement. Le texte qui contient les allusions les plus précises à ce curriculum est un autre texte Edubba, une composition publiée par Miguel Civil (1985) sous le nom « Edubba D » et répertorié aujourd'hui sous le nom « Dialogue $1 »^{23}$. Le texte est présenté ainsi par Civil :

Un de ces dialogues où des écoliers s'exercent à manier la langue sumérienne qui n'était pas leur langue maternelle. [...] Dans notre dialogue deux écoliers anonymes discutent de leur compétence en langue sumérienne en se vantant de leurs connaissances scolaires. Pour mieux décider qui l'emporte, on établit un long débat formel (a-da- $\min _{3}$ ) qui prend presque les trois quarts du texte et qui consiste en des tirades d'insultes plus ou moins vulgaires. C'est le premier quart du texte, qui décrit en détail l'apprentissage de la langue et de l'écriture, qui retiendra ici notre attention. (Civil $1985: 67$ ).

Les extraits suivants de ce «Dialogue 1 », dont le premier quart a été traduit par Civil 1985 à partir de 11 tablettes de Nippur, 1 tablette d'Ur, et 1 tablette d'origine inconnue, permet de saisir comment le curriculum est décrit dans les textes littéraires $:^{24}$

$[\ldots]$

2. Si tu es un écolier,

3. connais-tu le sumérien ?

4. Oui, je peux parler le sumérien.

5. $\quad[\mathrm{Tu}]$ es si jeune, comment peux-tu t'exprimer (si bien)?

6. J'ai écouté maintes fois les explications du maître,

\footnotetext{
${ }^{23}$ Le texte est numéroté 5.4.1 dans la base de données ETCSL, où il n'est ni transcrit, ni traduit (http://etcsl.orinst.ox.ac.uk/catalogue/catalogue5.htm).

${ }^{24}$ Les crochets « [...]» indiquent des passages du texte que j’ai sautés, et non des parties détruites.
} 
$[\ldots]$

12. J'ai (déjà) récité et écrit

11. les mots sumériens et akkadiens, depuis (le syllabaire) a-a me-me jusqu'à [...].

13. J'ai écrit toutes les lignes depuis (la liste de noms propres) Inanna-teš

14. jusqu'à (la série) $l_{u_{2}}=\check{s} \breve{u}$, même les formes désuètes.

$[\ldots]$

18. Je ne ferai rien de trop compliqué pour toi.

19. Même si l'on m'assigne (la série) $l_{2}=\breve{s} \bar{u}$, dans ma «tablette de main »,

20. Je peux donner l'ordre de six cents lignes avec $\operatorname{lu}_{2}$.

$[\ldots]$

28. Désormais, je peux m'appliquer aux tablettes, aux multiplications et aux bilans.

29. L'art de l'écriture, le placement des lignes, éviter les coupures, [...]

30. Mon maître a corrigé les belles paroles.

31. On doit se réjouir de la compagnie (des collègues)!

32. Je connais parfaitement mon art de l'écriture,

33. j'ai de la facilité pour tout.

34. Mon maître montre un signe,

35. j'en y ajoute plus d'un de mémoire.

36. Après avoir été à l'école jusqu'au temps prévu,

37. je suis à la hauteur du sumérien, de l'art de l'écriture, de la lecture des tablettes, du calcul des bilans.

38. Je peux parler sumérien!

39. Soit, (mais) le (sens du) sumérien t'est caché !

40. Je veux écrire des tablettes :

41. la tablette (des mesures) d'un gur d'orge jusqu'à six cents gur,

42. la tablette (des poids) d'un sicle jusqu'à vingt mines d'argent,

43. avec les contrats de mariage que l'on peut m'apporter,

44. les contrats de société, je peux choisir les poids contrôlés d'un talent,

45. la vente de maisons, de champs, d'esclaves,

46. les cautions en argent, les contrats de location de champs,

47. les contrats de culture des palmeraies, les [...],

48. même les contrats d'adoption, je sais écrire tout cela.

$[\ldots]$

Toutes les compositions citées dans le « Dialogue $1 »$ correspondent effectivement à des exercices scolaires attestés dans les tablettes scolaires produites par les écoliers : le syllabaire a-a me-me (ligne 11), les listes de noms propres (ligne 13), la liste de professions $\operatorname{lu}_{2}=\check{s} \bar{u}$ (lignes 14, 19 et 20), les listes métrologiques de mesures de capacités (ligne 41) et de poids (ligne 42), les modèles de contrats (lignes 43-48). De plus, ces textes sont cités dans l'ordre où ils étaient enseignés dans le curriculum (voir figure 1). La ligne 37 contient une description du contenu général de la formation de niveau élémentaire qui correspond au contenu des textes scolaires de Nippur connus : le sumérien, l'art de l'écriture, la lecture des tablettes, le calcul et les bilans. $\mathrm{Du}$ point de vue de la description du programme d'enseignement, le texte parait donc extrêmement réaliste. Il est cependant frappant, comme l'a noté Michalowski (2012 : 43), que les allusions au programme d'enseignement ne citent que les textes étudiés aux niveaux élémentaire et intermédiaire. Il n'est nulle part question, ni dans ce texte ni dans les autres textes $E d u b b a$, de littérature ou de problèmes de mathématiques de niveau avancé.

Les textes Edubba livrent donc des indices concordants avec d'autres sources sur certains aspects précis de l'enseignement, comme le curriculum. Les textes Edubba, à l'instar du « Dialogue 1 » cité ci-dessus, sont souvent écrits sous forme de dialogues, et témoignent de ce que l'enseignement de la langue sumérienne incluait l'écrit et l'oral, en particulier sous la 
forme de débats formels (Civil 1985 : 67). Il est sans doute révélateur de leur importance que de tels débats aient porté un nom en sumérien (voir citation de Civil 1985 : 67 au début de $§ 1.3$ ).

Nous avons souligné en introduction de ce chapitre que nous ne disposons que de très peu de sources d'information sur le contexte oral de l'enseignement. Les indices trouvés dans les textes littéraires sont donc précieux et peuvent apporter un éclairage unique. Nous allons, dans ce qui suit, nous appuyer plus encore sur ces textes pour essayer de détecter certains aspects de l'environnement d'oralité dans les écoles de scribes, en particulier en liaison avec l'apprentissage des mathématiques.

\subsection{La main du scribe a saisi la chanson}

Les textes littéraires de l'époque paléo-babylonienne comme l'Hymne Šulgi B évoqué en introduction semblent témoigner d'un processus où l'écrit remplace la mémoire dans la conservation de la tradition.

L’Hymne Šulgi B se présente comme une glorification du roi Šulgi par lui-même. Šulgi, deuxième souverain de la troisième dynastie d'Ur, a régné plus de quarante ans (env. 20942047). Les témoins de l'Hymne actuellement disponibles sont tous des tablettes scolaires d'époque paléo-babylonienne, trouvés principalement à Nippur. ${ }^{25}$ La date de sa composition est controversée. Jacob Klein estime, sur la base d'une analyse orthographique et grammaticale, que les hymnes dédiés à Šulgi ont été écrits de son vivant (Klein 1981 : 8, 27). Mais on ne peut exclure que l'hymne ait été rédigé à l'époque paléo-babylonienne par les maîtres des écoles dans un but d'enseignement, tout comme les textes Edubba (Michalowski 1986 : 839).

Le passage de l'Hymne Šulgi B suivant (lignes 308-3019) ${ }^{26}$ pourrait être interprété comme un écho de la transition de la tradition orale à la tradition écrite, phénomène dont la datation ne peut être déterminée puisque ni la date de composition de l'Hymne, ni celle des événements qu'il relate n'est identifiable.

308. Vers le sud, à Ur, j'ai dressé sur un sol pur

309. une « Maison de la Sagesse de Nisaba » pour l'écriture de mes chansons.

310. Vers le nord, à Nippur, j'en ai fondé une sur de solides fondations.

\footnotetext{
${ }^{25}$ Le texte est attesté sur env. 70 tablettes et fragments, dont 64 proviennent de Nippur, 4 d'Ur et 2 d'ailleurs selon ETCSL.

${ }^{26}$ Traduction de Piotr Michalowski (communication personnelle, août 2014 - voir note 13)

Southward, in Ur, I raised on consecrated ground

A "Nidaba's House of Wisdom" for the writing of my songs.

Northward, in Nippur I set one up on a firm foundation

And prayers that I had established in (temple) Ekur,

Should a scribe work on them, he will apply his hand.

Should a musician work on them, he will chant them aloud.

In places of learning may they never be changed ever,

In conservatories may they never to cease (to be leaned),

Now the wisdom that I accumulated should be just so:

Whatever is recited, throughout the wide world,

Whatever has been presented about me in full run of lyrics of my songs,

By An, Enlil, Utu and Inana, none of it is a lie - all of it is true!
} 
311. Les prières que j'ai établies dans ((le temple) Ekur,

312. puisse un scribe les fixer : il les saisira de sa main ;

313. puisse un musicien les fixer : il les chantera de sa voix.

314. Que dans l'Edubba, éternelles, elles restent pour toujours inchangées,

315. que dans les conservatoires, éternelles, elles ne cessent pour toujours d'être (enseignées).

316. Que la sagesse que j'ai accumulée dorénavant soit justement celle-ci :

317. quel que soit ce qui est récité de par le monde,

318. quelles que soient les collections de récits et de chants qui ont été présentées en mon honneur,

319. par An, Enlil, Utu et Inana, rien n'est un mensonge - tout cela est vrai !

Selon ce passage, ou tout au moins la traduction proposée ici, l'école, c'est-à-dire le lieu de Nisaba, la divinité des scribes, est l'endroit où la chanson est écrite (ligne 309). C'est la main du scribe qui saisit la chanson (ligne 312). Les lignes 317-319 sont assez difficiles à interpréter, mais semblent suggérer que les paroles à la gloire de Šulgi qui auront été saisies par écrit disent la vérité et assureront l'immortalité au roi (Cooper 2010 : 329). On peut entrevoir, derrière cette image de la main du scribe qui fixe par écrit la gloire de Šulgi pour la rendre immortelle, une métaphore du passage de l'oral à l'écrit de pans entiers des savoirs anciens, incluant la littérature sumérienne et les mathématiques.

\section{2 - « J'ai récité ma tablette » : mémorisation et duplicatas}

Quel était le rôle de la mémoire dans les processus d'apprentissage ? Le texte «Edubba $1 »$ évoqué plus haut, fait allusion au fait que l'apprentissage des listes lexicales et des tables mathématiques s'appuie sur la mémorisation.

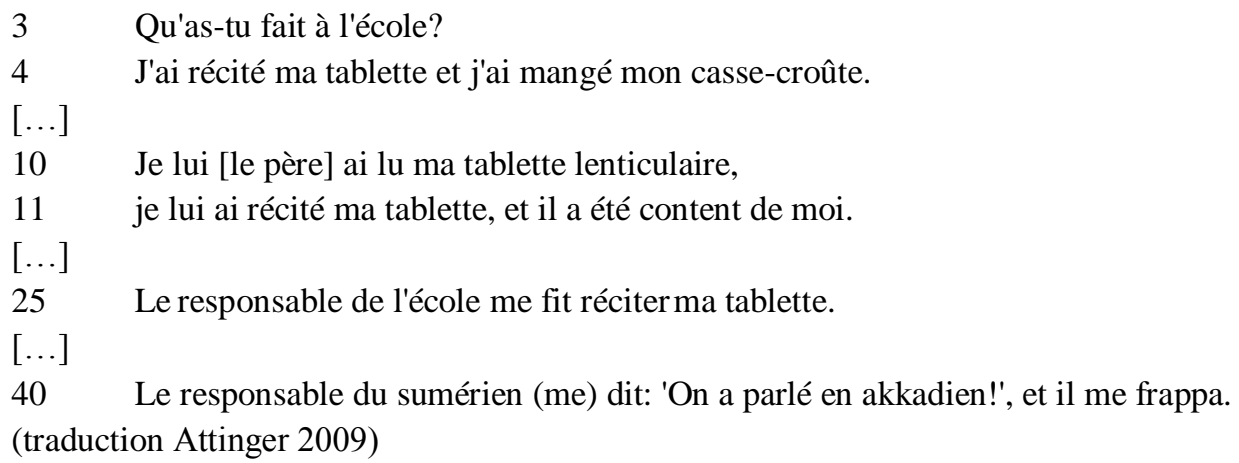

Si on en croit ces passages, les textes étaient assimilés par cœur et restitués par récitation. La langue de récitation n'est pas précisée, mais la ligne 40 souligne le fait que le sumérien est la langue de l'école, alors que la langue parlée en Mésopotamie à cette époque était l'akkadien. On peut supposer que les listes métrologiques et les tables de multiplication étaient mémorisées en sumérien, mais le texte ne le dit pas explicitement.

L'examen des productions des jeunes élèves scribes eux-mêmes nous en apprend un peu plus sur le processus de mémorisation et de restitution des textes, par récitation ou par écrit. Niek Velhuis et Paul Delnero se sont penchés sur les processus de reproduction des textes scolaires, le premier en s'intéressant principalement aux textes lexicaux, le deuxième aux textes 
littéraires, en observant les variantes d'un duplicata à l'autre et les erreurs dont ces variantes témoignent parfois. Pour Veldhuis comme pour Delnero, les listes lexicales et les textes littéraires utilisés dans l'enseignement étaient mémorisés et reproduits de mémoire. Cependant, pour d'autres auteurs, ces textes étaient dictés ou copiés sur un modèle. Veldhuis et Delnero se sont appuyés sur des travaux de neurosciences, notamment ceux de David Rubin, pour détecter les effets des phénomènes cognitifs sur les processus de reproduction des textes mémorisés. Veldhuis en a tiré la conclusion que les variantes nombreuses qu'on observe d'un duplicata à l'autre d'une liste lexicale peuvent refléter la flexibilité du fonctionnement de la mémoire (Veldhuis 1997: 132). Delnero (2012) a systématisé la démarche de comparaison des duplicatas utilisée par Veldhuis en s'intéressant aux dix textes littéraires ("Décade ») qui étaient étudiés par les jeunes scribes poursuivant des études avancées. Pour éviter les biais introduits par la diversité des pratiques d'enseignement, il a utilisé uniquement les sources de Nippur, et principalement les sources de la Maison F. Par ailleurs, il s'est appuyé plus largement que Veldhuis sur des études de neurosciences concernant le fonctionnement de la mémoire. Delnero montre que les erreurs qui émaillent les textes littéraires sumériens du Décade sont typiques de défaillances de mémoire, et c'est cette étude des erreurs qui lui permet de conclure que ces textes étaient reproduits de mémoire ${ }^{27}$.

Au-delà des conclusions de Veldhuis et de Delnero sur le processus de mémorisation, le point important pour notre enquête est le phénomène textuel qui en est la base. Il s'agit de la reproduction des textes à l'identique ou presque, pour certains à des centaines d'exemplaires. Ces duplicatas permettent de mettre en évidence de légères variantes, et éventuellement des erreurs révélatrices de phénomènes cognitifs. La question qui se pose alors est : quels sont les textes dont on connaît des duplicatas ? Pour ce qui est de l'apprentissage de l'écriture et de la langue sumérienne, les duplicatas sont observés pour la totalité du curriculum connu, en tout cas à Nippur, même si ce sont les listes lexicales écrites par des débutants qui sont le plus souvent reproduites. Pour ce qui est des mathématiques, la situation est légèrement différente : les listes et tables étudiées au niveau élémentaire sont attestées en de nombreux exemplaires, mais peu ou pas de duplicatas semblent avoir été produits lors des niveaux intermédiaires et avancés (voir figure 2). Les exercices mathématiques sont très stéréotypés, et se conforment à quelques modèles assez rigides, mais, pour autant qu'on puisse en juger par les sources connues, sont tous différents les uns des autres.

\footnotetext{
27 "Nearly all of the types of memory errors that occur in other corpora, and in particular the eight identified by Maguire and McGillivray described above, are attested in duplicatas of Sumerian literary compositions" (Delnero 2012: 198).
} 


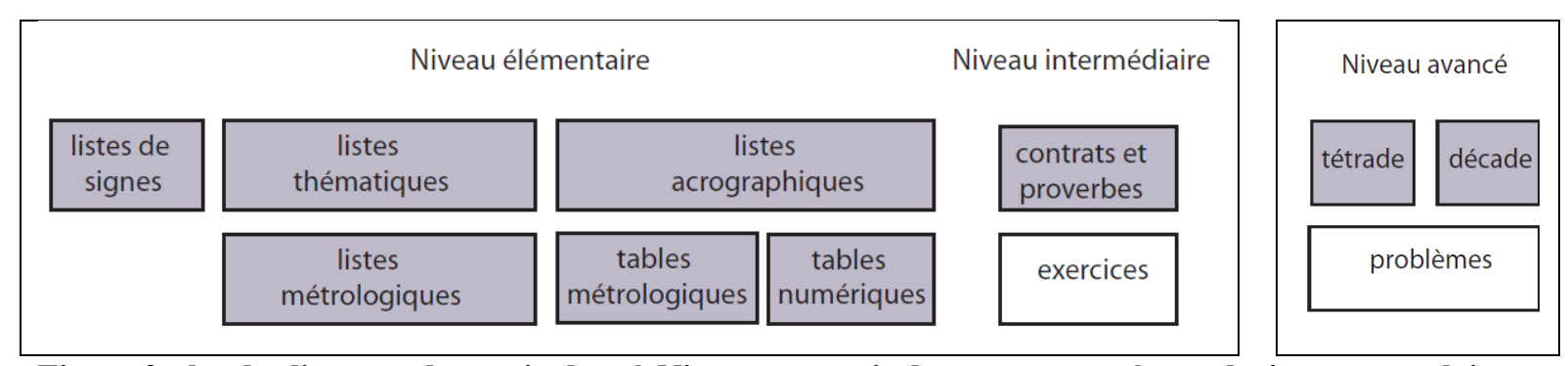

Figure 2 : les duplicatas et le curriculum à Nippur - en gris, les textes attestés en plusieurs exemplaires ; en blanc les textes attestés en un seul exemplaire

Cette asymétrie entre les curricula littéraire et mathématique est sans doute un symptôme de différenciations dans les processus d'apprentissage. L'apprentissage de la résolution des problèmes mathématiques ne relevait pas des mêmes méthodes que celui des structures grammaticales de la langue sumérienne. La question qui se pose pour les textes mathématiques de niveau élémentaire est, dès lors, celle de savoir si le processus d'assimilation des tables métrologiques et numériques était similaire à celui des listes lexicales. Plus précisément, on peut se demander, comme l'ont fait Veldhuis pour les listes lexicales et Delnero pour les textes littéraires, comment étaient reproduits les duplicatas : par copie sur un original ou « texte maître », sous la dictée, ou par cœur?

\section{3- « Je veux écrire la tablette des mesures d'un gur d’orge jusqu'à six cents gur » : mémoriser des textes mathématiques}

Une démarche similaire à celle de Veldhuis et Delnero, basée sur une comparaison des variantes entre duplicatas, pourrait-elle être entreprise pour les textes mathématiques de niveau élémentaire ? Les listes et tables métrologiques et tables numériques de Nippur présentent une certaine stabilité, et l'ensemble des variantes et erreurs ne fournit qu'un matériel réduit. La logique interne qui gouverne les textes mathématiques est sans doute une des raisons de la stabilité des listes et tables métrologiques et numériques de niveau élémentaire. Pour ce qui est des textes de Nippur, les erreurs que j'ai constatées sont essentiellement limitées à des éléments (clous ou chevrons) omis ou surnuméraires. Il arrive aussi que des items soient omis ${ }^{28}$. On note également des variantes dans la granularité des listes et tables métrologiques, c'est-à-dire l'introduction de mesures intermédiaires entre deux mesures données. Des exemples de variations de granularité sont données dans le tableau 1, qui présente trois extraits de liste métrologique de mesures de capacité provenant de tablettes scolaires de Nippur. On constate que les choix des scribes en ce qui concerne la granularité de la liste sont différents : aucune fraction de gin n'apparaît dans le premier exemple, des fractions de gin apparaissent entre 1 gin et 2 gin dans le deuxième exemple, et des fractions de gin apparaissent entre 1 gin et 2 gin, 2 gin et 3 gin dans le troisième exemple.

\footnotetext{
${ }^{28}$ Ce sont essentiellement ces types d'erreurs que relève Hilprecht, par exemple, dans sa copie de la tablette CBS $11340+$ (voir fig. 4).
} 


\begin{tabular}{|c|c|c|}
\hline $\begin{array}{l}\left.\text { HS } 249 \text { (TMH } 8 \mathrm{n}^{\circ} 3\right) \\
\text { face, col. } i\end{array}$ & $\begin{array}{l}\left.\text { HS 239+ (TMH } 8 \mathrm{n}^{\circ} 6\right) \\
\text { rev., col. } i\end{array}$ & $\begin{array}{l}\text { HS } 1790\left(\mathrm{TMH} 8 \mathrm{n}^{\circ} 13\right) \\
\text { rev., col. } i\end{array}$ \\
\hline 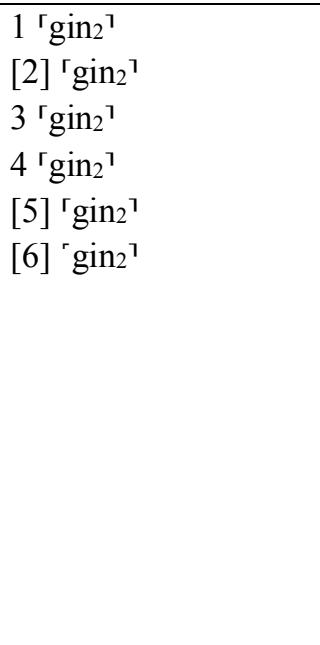 & $\begin{array}{l}1 \operatorname{gin}_{2} \mathrm{s̆e} \\
11 / 3 \operatorname{gin}_{2} \\
11 / 2 \operatorname{gin}_{2} \\
12 / 3 \operatorname{gin}_{2} \\
15 / 6 \operatorname{gin}_{2} \\
2 \operatorname{gin}_{2} \\
3 \operatorname{gin}_{2} \\
4 \operatorname{gin}_{2} \\
5 \operatorname{gin}^{\top}{ }^{7} \\
6\left[\operatorname{gin}_{2}\right]\end{array}$ & $\begin{array}{l}\text { [1] } \operatorname{gin}_{2} \mathrm{s̆e} \\
\text { [1] } 1 / 3 \operatorname{gin}_{2} \\
\text { [1] } 1 / 2 \operatorname{gin}_{2} \\
12 / 3 \operatorname{gin}_{2} \\
15 / 6 \operatorname{gin}_{2} \\
2 \operatorname{gin}_{2} \\
21 / 3 \operatorname{gin}_{2} \\
21 / 2 \operatorname{gin}_{2} \\
22 / 3 \operatorname{gin}_{2} \\
25 / 6 \operatorname{gin}_{2} \\
3 \operatorname{gin}_{2} \\
4 \operatorname{gin}_{2} \\
\text { [5] } \operatorname{gin}_{2} \\
\text { [6] }{ }^{\top} \operatorname{gin}_{2}{ }^{1}\end{array}$ \\
\hline
\end{tabular}

Tableau 1 : extraits de listes métrologiques de mesures de capacités (intervalle 1 gin - 6 gin)

Dans quelle mesure les variantes de ce type relèvent-elles des choix intentionnels ? Pour répondre à cette question, on peut essayer d'appliquer les critères définis par Delnero permettant de distinguer une variante intentionnelle d'une faute, ou plutôt ici d'un oubli.

... a variant has only been classified as an error if it meets all of the following conditions:

1) the variant only occurs in one of the preserved sources for the line;

2) the source in which the variant occurs does not contain multiple variants of the same type;

3) variants of the same type are rare in Sumerian literary sources; and

4) whenever distinguishable, the form is overtly incorrect and differs substantially from the form that occurs in the other preserved sources. (Delnero 2012: 200)

Dans le cas des listes et tables métrologiques, les variations de granularité sont correctes d'un point de vue mathématique; elles sont relativement systématiques dans un texte donné : la présence de mesures intermédiaires entre deux mesures données n'est pas ponctuelle, mais apparaît comme une tendance sur l'ensemble du texte ; elles ne sont pas exceptionnelles : certaines listes ou tables métrologiques présentent une granularité plus fine que les autres. Appliqués à notre exemple, les critères de Delnero permettent de conclure que les variantes de granularité sont intentionnelles, et reflètent des choix délibérés.

\section{Un exemple provenant de Nippur}

Il convient toutefois de noter que l'absence ou la rareté des erreurs peut être en ellemême une information, et refléter la façon dont le texte a été reproduit, comme l'exemple des tables de multiplication le montre. Considérons par exemple une grande tablette multi-colonnes 
(type I selon la nomenclature des assyriologues ${ }^{29}$ ) contenant toutes les tables numériques, comme la tablette Ist Ni 2733.

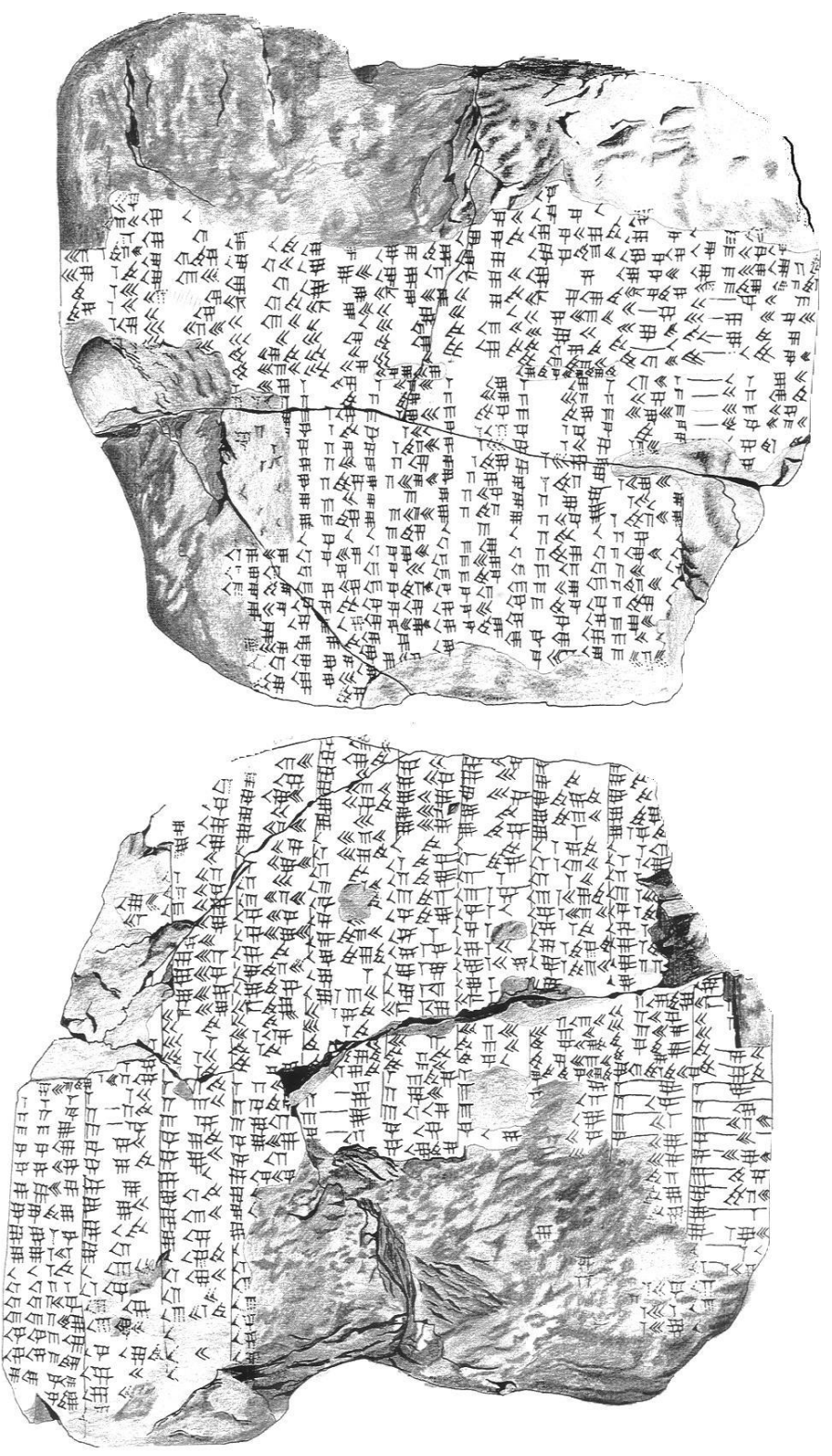

Figure 3 : Ist Ni 2733, Musée archéologique d'Istanbul (copie Proust 2007: pl. 5-6) ${ }^{30}$

\footnotetext{
${ }^{29}$ La typologie des tablettes scolaires a fait l'objet de nombreuses études (voir notamment Veldhuis 1997 : 2941 et Delnero $2010: 54$ et la bibliographie fournie par ces publications). A Nippur, on distingue quatre types de tablettes qui étaient utilisées au niveau élémentaire : les tablettes de type I sont de grandes tablettes multi-colonnes contenant un texte continu commençant sur la face et se terminant sur le revers (voir Ist Ni 2733, fig. 3) ; les tablettes de type II contiennent sur la face un court extrait reproduit une ou deux fois, et sur le revers un long extrait d'un autre texte que celui qu'on trouve sur la face ; les tablettes de type III sont de petites tablettes uni-colonne contenant un court extrait (typiquement, dans le cas des mathématiques, une table de multiplication) ; les tablettes de type IV sont des tablettes lenticulaires ou carrées contenant seulement quelques lignes sur la face (pour ce qui est des mathématiques, ce type de tablette n'était que très peu utilisé dans les niveaux élémentaires et n'apparaît que pour des enseignements plus avancés dans le curriculum).

${ }^{30}$ Cette copie contient certainement des erreurs. Mais la plupart d'entre elles ne sont pas le fait du jeune scribe de Nippur qui l'a écrite, elles proviennent du copiste moderne !
} 
Il est remarquable qu'un texte qui contient plus de 900 résultats (inverses, produits et carrés) ne contienne que peu d'erreurs. Pourtant, une reproduction d'après un modèle d'un tel ensemble de tables par un copiste ne les connaissant pas par cœur n'aurait pu que générer de nombreuses erreurs. S'il s'agit bien d'un ensemble de tables restitué de mémoire, il semblerait alors que la mémorisation des tables numériques ne fonctionnât pas de la même façon que la mémorisation d'un texte littéraire. Le faible nombre d'erreurs serait, dans le cas des tables numériques, un symptôme de mémorisation. Delnero a montré au contraire que dans le cas des textes littéraires, c'est le grand nombre d'erreurs qui est un symptôme de mémorisation (Delnero 2012 : 207).

Cependant, le faible nombre d'erreurs dans une table comme Ist Ni 2733 n'est pas un indice suffisant pour conclure que ces tables étaient mémorisées. En effet, certaines tables comportent beaucoup plus d'erreurs, en particulier celles qui sont écrites sur des tablettes de type II (voir, fig. 4 ci-après la copie de la tablette CBS 114340+11402 par Hilprecht, qui a noté toutes les erreurs qu'il a rencontrées). De plus, un copiste ne connaissant pas les tables par cœur, mais maîtrisant bien les processus qui les engendre, aurait sans doute été capable de contrôler une copie reproduite d'après un modèle et d'éviter les erreurs. Un autre indice vient cependant corroborer l'hypothèse de la mémorisation. Les tables métrologiques et numériques étaient des outils utilisés régulièrement par les scribes professionnels, comme les maîtres des Edubba ou des administrateurs, dont les activités incluaient sans doute la pratique du calcul sexagésimal positionnel. Cependant, ces tables ne sont attestées à Nippur que sur des tablettes typiquement scolaires (types I, II ou III). Les tables métrologiques et numériques ne semblent pas avoir écrites en tant que texte de référence pour des professionnels, tout au moins pour ce qui concerne Nippur ${ }^{31}$. Bien qu'il soit hasardeux de tirer des conclusions d'une absence de source, on peut envisager l'explication suivante : si les tables métrologiques et mathématiques ne sont attestées que sous forme de textes scolaires, c'est parce que ces textes devenaient inutiles après la formation élémentaire du fait qu'ils avaient été mémorisés ${ }^{32}$.

Il est donc probable que la mémorisation jouait un grand rôle dans la formation mathématique élémentaire. Mais peut-on en savoir plus sur le processus de mise en mémoire, et sur les processus de restitution des données mémorisées ? De nombreuses études ont attiré l'attention sur l'intérêt de l'observation des tablettes de type II pour comprendre les méthodes d'apprentissage pratiquées dans les écoles. Sur la face de ce type de tablettes, on trouve un modèle, sans doute écrit de mémoire par le maître, et une ou deux copies, probablement réalisées par un jeune scribe en formation. A ce stade, le texte n'est pas encore mémorisé par l'élève. Veldhuis a montré que dans le même temps, alors qu'il était en train d'assimiler de

\footnotetext{
${ }^{31}$ Le tableau était peut-être différent de celui qui est dépeint ici dans d'autres sites, où on a trouvé des tables métrologiques ou numériques écrites sur cylindre ou prisme (voir par exemple le prisme AO 8865 dans Proust 2005, le prisme YBC 2392 et le cylindre VA 2596 dans Chambon et Robson 2011).

${ }^{32}$ De la même façon aujourd'hui, les tables de multiplication qui figurent au dos des cahiers de brouillon des écoliers modernes disparaissent des documents mathématiques destinés à des étudiants plus avancés ou des professionnels. En revanche, les tables trigonométriques ou de logarithmes qui étaient utilisées quotidiennement pour le calcul avancé avant la diffusion des calculatrices de poche n'étaient pas mémorisées et figuraient dans les bibliothèques des ingénieurs.
} 
nouveaux textes, l'étudiant devait s'exercer à restituer par écrit les listes lexicales mémorisées dans les étapes antérieures de son apprentissage. Ces exercices de contrôle de la mémorisation se trouvent sur le revers des tablettes de type II. Le même processus de mise en mémoire s'appliquait aux mathématiques, comme le montre la répartition des tables de multiplication sur les tablettes de type II à Nippur. ${ }^{33}$

\begin{tabular}{|l|l|l|l|}
\hline $\begin{array}{l}\text { Numéro de } \\
\text { musée }\end{array}$ & $\begin{array}{l}\text { Contenu de } \\
\text { la face }\end{array}$ & Contenu du revers & Publication \\
\hline $\begin{array}{l}\text { CBS 11340 + } \\
\text { CBS 11402 }+ \\
\text { UM 29-16-752 }\end{array}$ & $\begin{array}{l}\times 45 \text { (modèle } \\
\text { et copie) }\end{array}$ & inverses, $\times 50 ;$ inverses, $\times 50 ;$ inverses, $\times 50$ & BE \\
\hline Ni 10119 & $\times 44: 26: 40$ & inverses et $\times 50$ (répétées plusieurs fois) \\
\hline Ni 2726 & $\times 44: 26: 40$ & inverses; $\times 50 ; \times 45 ; \times 44: 26: 40$ & TMN \\
\hline Ni 10109 & $\times 30$ & inverses, $\times 50 ; \times 45$ (répétées plusieurs fois) & TMN \\
\hline HS 205 & $\times 24$ & $\times 30 ; \times 25 ;$ inverses & TMN \\
\hline Ni 3714 & $\times 22: 30$ & $\times 40 ; \times 36 ; \times 30 ; \times 25$ & TMH 8 \\
\hline Ni 3509+3509 & $\times 22: 30$ & $\times 30 ; \times 25$ & TMN \\
\hline Ni 10221 & $\times 18$ & $\times 30 ; \times 25 ; \times 24 ; \times 22: 30$ & TMN \\
\hline Ni 3786 & $\times 16: 40$ & inverses,$\times 45$ & TMN \\
\hline HS 203 & $\times 16: 40$ & $\begin{array}{l}\text { inverses; } \times 50 ; \times 45 ; \times 44: 26: 40 ; \times 40 ; \times 36 ; \times 30 ; \times 25 ; \times 24 ; \\
\times 22: 30 ; \times 20 ; \times 18 ; \times 16: 40 ; \text { inverses }\end{array}$ & TMH 8 \\
\hline CBS 8161 & $\times 16: 40$ & $\times 24 ; \times 20 ; \times 18$ & CDLI \\
\hline HS 207 & $\times 15$ & $\times 24 ; \times 22: 30$ & TMH 8 \\
\hline Ni 5393 & $\times 12$ & $\times 16 ; \times ?$ & TMN \\
\hline CBS 6095 & $\times 3: 45$ & $\times 45 ; \times 44: 26: 40 ; \times 40 ; \times 36 ; \times 30 ; \times 25 ; \times 24 ; \times 22: 30 ; \times 20 ; \times 18 ;$ & MCT \\
\hline
\end{tabular}

Tableau 2 : les tablettes de type II contenant des tables de multiplication sur la face et le revers - sélection parmi les sources de Nippur

L'ordre d'apprentissage des tables numériques était toujours le même : inverses ; $\times 50$; $\times 45 ; \times 44: 26: 40 ; \times 40 ; \times 36 ; \times 30 ; \times 25 ; \times 24 ; \times 22: 30 ; \times 20 ; \times 18 ; \times 16: 40 ; \times 16 ; \times 15 ;$ $\times 12: 30 ; \times 12 ; \times 10 ; \times 9 ; \times 8: 20 ; \times 8 ; 7: 30 ; \times 7: 12 ; \times 7 ; \times 6: 40 ; \times 6 ; \times 5 ; \times 4: 30 ; \times 4 ; \times 3: 45 ;$ $\times 3: 20 ; \times 3 ; \times 2: 30 ; \times 2: 24 ; \times 2 ; \times 1: 40 ; \times 1: 30 ; \times 1: 20 ; \times 1: 15 ; \times$ carrés $^{34}$. C'est cet ordre immuable qu' on trouve dans tous les textes qui contiennent plusieurs tables de multiplication, par exemple dans les tablettes de type I (voir la tablette Ist Ni 2733, fig. 3). Lorsqu'une nouvelle table était étudiée par un jeune scribe, le modèle était écrit par le maître, et l'élève s'exerçait à reproduire ce modèle une ou deux fois. Par exemple, sur la face de la tablette CBS $114340+11402$, on voit la table de multiplication par 45 écrite par un maître et reproduite par un élève (figure 4). Sur le revers de cette même tablette, on trouve la liste de toutes les tables numériques mémorisées antérieurement, c'est-à-dire depuis le début de la série jusqu'à la table

\footnotetext{
${ }^{33}$ Voir aussi Robson $2002: 339$; Proust $2007:$ ch. 5.

${ }^{34}$ Les nombres utilisés dans les tables de multiplication étaient écrit en notation sexagésimale positionnelle. Ici, en particulier dans cette liste de nombres principaux des tables de multiplication et dans le tableau 2, la transcription des nombres reflète cette notation sexagésimale positionnelle. Le séparateur de chiffres sexagésimaux adopté ici est le signe «: », qui évoque le séparateur entre secondes, minutes et heures utilisé de nos jours dans la notation sexagésimale, par exemple sur les écrans montres ou sur les compteurs de vidéo. Notons que pour représenter ce séparateur dans les publications modernes, il est plus souvent utilisé le point ou la virgule.
} 
de 45 , soit, en l'occurrence, les tables inverses et $\times 50$ selon l'ordre fixe immuable. Cette séquence étant brève, l'espace disponible sur le revers a permis de la reproduire trois fois. La tablette HS 203 contient sur la face une table de 16:40, et sur le revers la liste de toutes les tables antérieures selon l'ordre du curriculum (voir tableau 2); la série des tables mémorisées antérieurement à celle de 16:40 étant plus longue, elle n'a pas pu pas être inscrite plusieurs fois sur le revers. Le tableau 2 montre comment ce processus apparaît dans d'autres tablettes de type II.

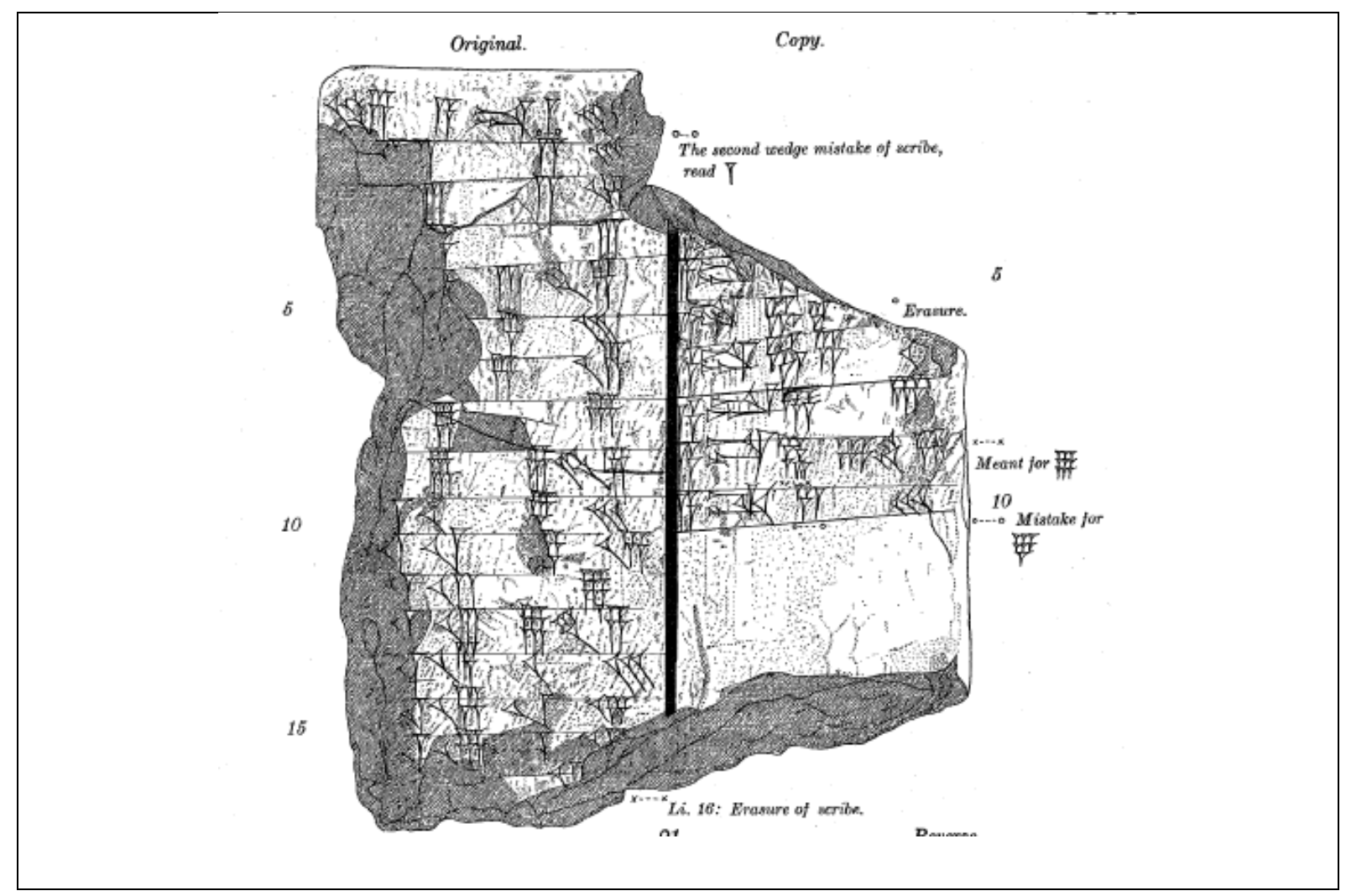




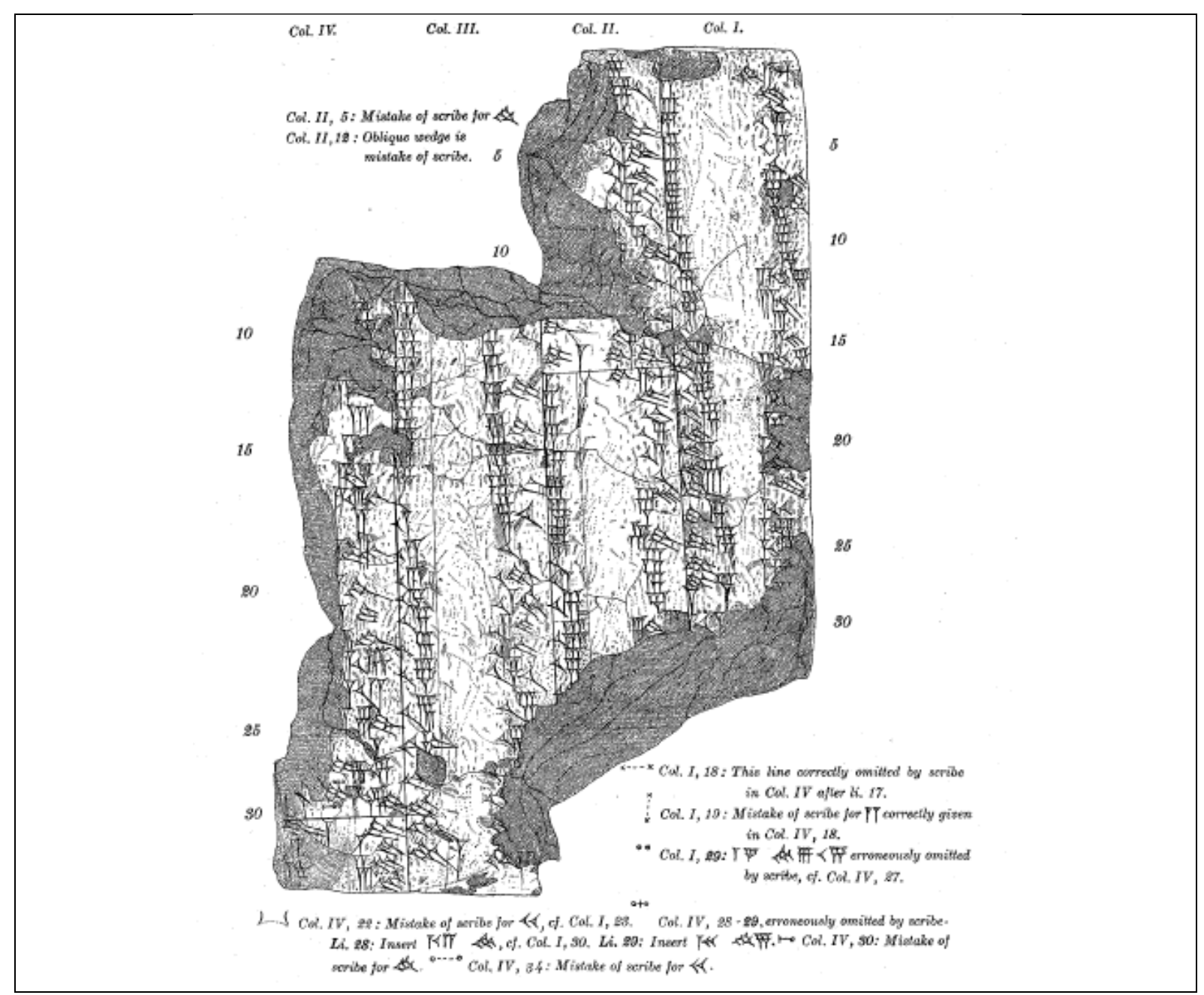

Figure 4 : copie des fragments CBS 11340+11402 par Hilprecht (1906 : pl. 10-11). Les erreurs relevées par Hilprecht sont signalées en marge de la copie.

Cette structure des textes sur les tablettes de type II explique le phénomène observé par Veldhuis (1997 : 36) pour les listes lexicales : les statistiques concernant le nombre d'attestation des textes ou portions de textes dans les tablettes de type II montrent que les parties appartenant au début des séries (par exemple, les premières de l'ensemble des tables de multiplication) sont beaucoup plus fréquentes que celles qui appartiennent à la fin des séries ${ }^{35}$.

Dans les sources de Nippur, la mémorisation apparaît comme un processus à la fois écrit et oral, qui intègre la copie, la restitution par écrit (selon les tablettes de type II), et la récitation (selon les « témoignages » des textes littéraires).

\section{Un exemple provenant de Kiš}

Si on quitte Nippur, on trouve un autre cas intéressant avec la tablette Ashm 1931.137 provenant de Kiš en Mésopotamie centrale. ${ }^{36}$ Cette grande tablette multi-colonnes de type I

\footnotetext{
${ }^{35}$ Voir aussi Robson 2001 : 49; Proust 2007 : 271. En revanche, la répartition des tables de multiplication est à peu près régulière dans les tablettes de type I ou III (Proust $2007: 271$, tableau 3).

${ }^{36}$ Cette tablette a été publiée par Eleanor Robson, qui donne une description détaillée des erreurs contenues dans le texte (Robson 2004: 35).
} 
contient un ensemble de listes de mesures de capacité, de poids et de surface. Il est possible que cette tablette ait été le travail d'un étudiant qui terminait le niveau élémentaire de la formation et exécutait une sorte d'œuvre récapitulative ou d'examen. Cet « examen » contient des erreurs qui touchent des pans entiers du texte, et ne semble donc pas très réussi.

On trouve dans ce texte trois séquences fautives, qui correspondent à trois types différents d'erreurs. La première séquence fautive se trouve à la fin de la colonne iii de la face (tableau 3).

\begin{tabular}{|c|c|c|c|}
\hline \multicolumn{3}{|c|}{$\begin{array}{l}\text { Translitération (traduction) } \\
\text { Parties fautives en gras }\end{array}$} & Séquence correcte \\
\hline \multicolumn{4}{|c|}{ Face col. iii } \\
\hline $\begin{array}{l}\cdots \\
1 \text { (geš’u) }\end{array}$ & še gur & (600 gur) & Idem \\
\hline 2(geš'u) & še gur & $(2 \times 600$ gur $)$ & Idem \\
\hline 3(geš’u) & še gur & $(3 \times 600$ gur $)$ & Idem \\
\hline 4(geš’u) & še gur & $(4 \times 600$ gur $)$ & Idem \\
\hline 5 (geš’u) & še gur & $(5 \times 600$ gur $)$ & Idem \\
\hline 6(geš’u) & še gur & $(6 \times 600$ gur $)$ & $\varnothing$ \\
\hline 7 (geš’u) & še gur & $(7 \times 600$ gur $)$ & $\varnothing$ \\
\hline 8(geš’u) & še gur & $(8 \times 600$ gur $)$ & $\varnothing$ \\
\hline 9(geš’u) & še gur & $(9 \times 600$ gur $)$ & $\varnothing$ \\
\hline
\end{tabular}

Tableau 3 : première séquence fautive de la tablette Ashm 1931.137

La séquence 600 gur / $2 \times 600$ gur / 3×600 gur / 4×600 gur / 5×600 gur $(1$ (geš’u $)=600 ; 1$ gur $=$ env. 300 litres), qui est correcte, est poursuivie avec une séquence additionnelle de quatre items qu'on ne trouve dans aucune autre liste métrologique connue : 6×600 gur / 7×600 gur / 8×600 gur / 9×600 gur. Les signes numériques de cette séquence additionnelle ne semblent du reste pas très bien formés, trahissant peut-être un doute dans l'esprit de leur auteur. Cette séquence additionnelle répond à une certaine logique, puisque le scribe a été enclin à poursuivre le compte $1,2,3,4,5(\times 600$ gur) pour l'étendre à $6,7,8,9(\times 600$ gur $)$. Cependant, cette logique témoigne d'une incompréhension du système numérique (ledit « système $\mathrm{S}$ ») utilisé dans tous les autres textes de l'époque paléo-babylonienne pour compter les unités de mesure de capacité gur. Ce système peut être représenté de la façon suivante :

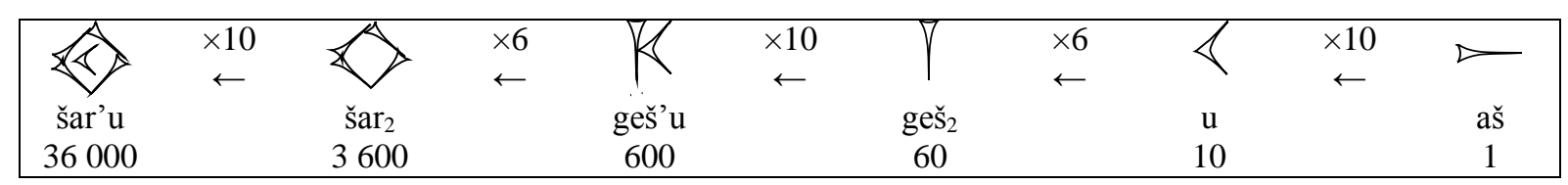

Figure 5 : système $S$

Selon ce système numérique, le nombre 3600 ne s'écrit pas 6(geš'u), c'est-à-dire six fois le

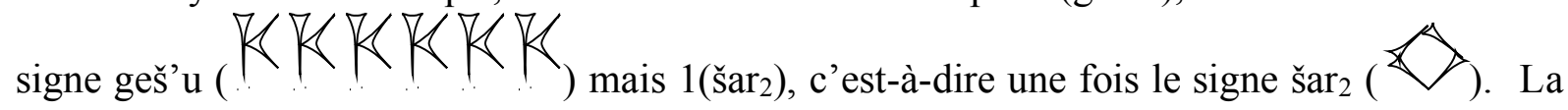
séquence correcte apparaît au début de la colonne suivante avec $1 \times 3600$ gur / 2×3600 gur / 


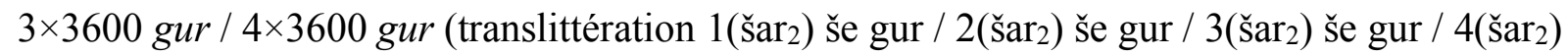
še gur).

Séquence fautive : compte des geš'u tel qu'on peut le deviner à la fin de la colonne iii

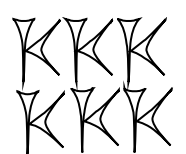

$6($ geš’u)

$6 \times 600$

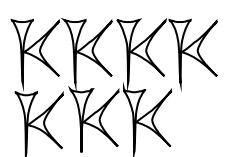

7 (geš'u)

$7 \times 600$

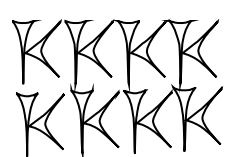

$8($ geš’u)

$8 \times 600$

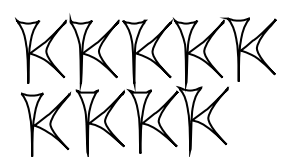

$9($ geš'u)

$9 \times 600$

Séquence correcte : compte des šar'u correcte qui aurait dû apparaître à la fin de la colonne iii (et, en fait, apparaît au début de la colonne iv)

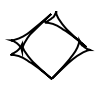

$1\left(\breve{s a r}_{2}\right)$

3600

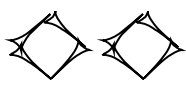

$2\left(\breve{s}_{2} r_{2}\right)$

$2 \times 3600$

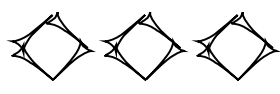

$3\left(\check{\mathrm{sar}}_{2}\right)$

$3 \times 3600$

etc.

Figure 6 : première séquence fautive dans la tablette Ashm 1931.137

Il est clair qu'il s'agit bien d'une erreur, et qu'une telle erreur ne reflète ni une faute sous la dictée, car la verbalisation de 6 (geš'u) ne devait aucunement ressembler à celle de 1(šar 2 ), ni une faute de copie, car la séquence fautive, entièrement inventée, a un aspect visuel qui ne peut être confondu avec celui de la séquence correcte. Le scribe a simplement extrapolé selon sa logique propre la liste existante, en ajoutant une séquence qui fait appel à une notation fautive en regard du système sexagésimal en vigueur. On a donc bien, avec cette erreur, une trace incontestable de reproduction de mémoire d'une liste métrologique.

La deuxième séquence fautive est la répétition de la séquence $3600 \mathrm{gur} / 2 \times 3600 \mathrm{gur}$ / 3×3600 gur / 4×3600 gur / 5×3600 gur / 6×3600 gur / 7×3600 gur / 8×3600 gur / 9×3600 gur. Là encore, on imagine difficilement qu'une séquence aussi longue ait pu être répétée par erreur sous la dictée ou en reproduisant un modèle ${ }^{37}$.

\footnotetext{
${ }^{37}$ Les répétitions d'un mot, ou dittographies, sont en revanche caractéristiques des textes copiés sur un modèle
} (Delnero 2012 : 190, 203-4, 207). 


\begin{tabular}{|c|c|c|c|c|c|}
\hline \multicolumn{3}{|c|}{$\begin{array}{l}\text { Translitération (traduction) } \\
\text { Parties fautives en gras }\end{array}$} & \multicolumn{3}{|c|}{ Séquence correcte } \\
\hline \multicolumn{3}{|l|}{ Face col. iv } & & & \\
\hline $1\left(\check{s} a r_{2}\right)$ & še gur & (3 600 gur $)$ & Idem & & \\
\hline $2\left(\right.$ s̆ar $\left._{2}\right)$ & še gur & $(2 \times 3600$ gur $)$ & Idem & & \\
\hline $3\left(\breve{s a r}_{2}\right)$ & še gur & $(3 \times 3600$ gur $)$ & Idem & & \\
\hline $4\left(\right.$ šar $\left._{2}\right)$ & še gur & $(4 \times 3600$ gur $)$ & Idem & & \\
\hline $5\left(\right.$ s̆ar$\left._{2}\right)$ & še gur & $(5 \times 3600$ gur $)$ & Idem & & \\
\hline $6\left(\mathrm{s̆ar}_{2}\right)$ & še gur & $(6 \times 3600$ gur $)$ & Idem & & \\
\hline $7\left(\right.$ šar $\left._{2}\right)$ & še gur & $(7 \times 3600$ gur $)$ & Idem & & \\
\hline $8\left(\right.$ šar $\left._{2}\right)$ & še gur & $(8 \times 3600$ gur $)$ & Idem & & \\
\hline $9\left(\right.$ šar $\left._{2}\right)$ & še gur & $(9 \times 3600$ gur $)$ & Idem & & \\
\hline 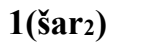 & še gur & (3 600 gur) & $\varnothing$ & & \\
\hline $2\left(\check{s ̆ a r}_{2}\right)$ & še gur & $(2 \times 3600$ gur $)$ & $\varnothing$ & & \\
\hline 3( šar 2$)$ & še gur & $(3 \times 3600$ gur $)$ & $\varnothing$ & & \\
\hline $4\left(\right.$ šar $\left._{2}\right)$ & še gur & $(4 \times 3600$ gur $)$ & $\varnothing$ & & \\
\hline 5( šar $\left._{2}\right)$ & še gur & $(5 \times 3600$ gur $)$ & $\varnothing$ & & \\
\hline 6(行ar 2$)$ & še gur & $(6 \times 3600$ gur $)$ & $\varnothing$ & & \\
\hline 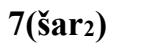 & še gur & $(7 \times 3600$ gur $)$ & $\varnothing$ & & \\
\hline 8(šar 2$)$ & še gur & $(8 \times 3600$ gur $)$ & $\varnothing$ & & \\
\hline 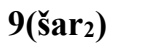 & še gur & $(9 \times 3600$ gur $)$ & $\varnothing$ & & \\
\hline 1 (šar’u) & še gur & (36000 gur) & Idem & & \\
\hline 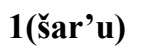 & še gur & (36 000 gur) & $1($ šar'2u) & še gur & $(2 \times 36000 \mathrm{gur})$ \\
\hline 1(šar’u) & še gur & (36000 gur) & 1(šar’3u) & še gur & $(3 \times 36000 \mathrm{gur})$ \\
\hline 1(šar’u) & še gur & (36000 gur) & $1($ šar'4u) & še gur & $(4 \times 36000 \mathrm{gur})$ \\
\hline <5(吕’’u) & še gur $>$ & & $1($ šar'5u) & še gur & $(5 \times 36000 \mathrm{gur})$ \\
\hline $1\left(\check{s a r}_{2}\right) \mathrm{gal}$ & še gur & $(36000 \times 60$ gur $)$ & Idem & & \\
\hline
\end{tabular}

Tableau 4 : deuxième et troisième séquences fautives de la tablette Ashm 1931.137

La troisième séquence fautive se trouve à la fin de la colonne $i v$ de la face. Le scribe a répété quatre fois le même item et écrit $36000 \mathrm{gur} / 36000 \mathrm{gur} / 36000 \mathrm{gur} / 36000 \mathrm{gur}$ au lieu de décliner correctement le compte des šar'u (36 000) en cinq items $36000 \mathrm{gur} / 2 \times 36000$ gur / 3×36000 gur / 4×36000 gur / 5×36 000 gur. Sur le plan graphique, le compte des šar'u (36 000) consistait soit à répéter autant de fois que nécessaire le signe šar'u, soit à introduire des signes 10 ( $\ll$ ) à l'intérieur du signe šar 2 (voir figure 7). C'est cette dernière option qui est adoptée ailleurs dans le présent texte, et qui donc aurait dû être adopté dans cette séquence. 
Séquence fautive : compte des šar'u qui apparait dans le texte

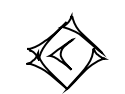

1(šar'u)

36000

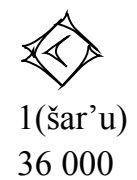

36000
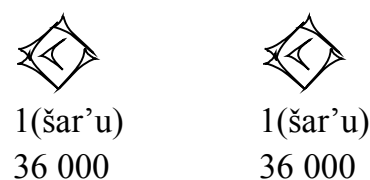

Séquence correcte : compte des šar'u qui aurait dû apparaître dans le texte

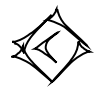

1(šar'u)

36000

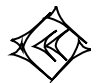

(šar'2u)

$2 \times 36000$

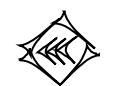

(šar'3u)

$3 \times 36000$

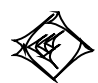

(šar' $3 u$ )

$4 \times 36000$

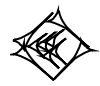

(šar' $5 \mathrm{u}$ )

$5 \times 36000$

Figure 7 : graphies dans la deuxième séquence fautive dans la tablette Ashm 1931.137

Peut-être le scribe a-t-il oublié comment le compte des šar'u était noté, ou bien a-t-il préparé la séquence en notant tous ses éléments fixes ( puis a oublié de compléter ensuite en ajoutant les signes $10(\varangle)$ nécessaires à l'intérieur du signe šar' ${ }^{38}$. Mais dans ce dernier cas, le scribe aurait dû écrire cinq fois le même item et non pas quatre fois. Il est donc probable qu'il s'agit plutôt d'une défaillance de mémoire.

Dans les trois cas, les erreurs dans la tablette Ashm 1931.137 semblent révéler des défaillances dans le processus de mémorisation ou de restitution par écrit d'un texte mémorisé. Le cas des erreurs dans la tablette Ashm 1931.137 appelle deux remarques sur le processus de transmission des liste métrologiques et le problème de la source originale dans les chaînes de transmission. Si les listes métrologiques avaient été transmises par la tradition écrite et n'étaient parvenues jusqu'à nous que par ce canal, les erreurs détectées dans une source archéologique telle que la tablette Ashm 1931.137 auraient sans doute été corrigées lors des éditions successives, et ces corrections auraient alors effacé les traces du contexte de mémorisation qui a probablement vu naître ce texte. Notons également que la liste métrologique écrite sur la tablette Ashm 1931.137 n'a pas été créée par l'auteur de cette tablette, puisque cette liste, avec des variantes, est attestée sur de très nombreux duplicatas, pour certains probablement plus anciens, provenant de Nippur, et d'autres sites. Les listes et tables métrologiques et numériques, attestées essentiellement par des sources datant de l'époque paléo-babylonienne, sont ellesmêmes le produit d'une transmission écrite combinée avec une tradition orale probablement encore plus ancienne.

\section{Conclusion : écoles et oralité}

Revenons à la question posée au début de ce chapitre : pourquoi les textes scolaires apparaissent massivement dans nos sources à l'époque paléo-babylonienne, alors que de

\footnotetext{
${ }^{38}$ Ce type d'oubli est fréquent de nos jours lorsqu'on utilise l'outil « copier/coller » des logiciels de traitement de texte pour reproduire une séquence répétitive.
} 
nombreux indices laissent penser que les écoles de scribes ont existé bien avant ? L'absence de sources scolaires datant d'Ur III pourrait refléter des changements dans la nature et le fonctionnement des écoles ${ }^{39}$. Le premier de ces changements est la place de plus en plus grande de la tradition écrite. Pour ce qui est des mathématiques, il est probable que la plupart des listes et tables métrologiques et numériques qui fournissaient l'essentiel de l'apprentissage des mathématiques au niveau élémentaire des écoles paléo-babyloniennes existaient déjà dans les époques antérieures. De ces témoins plus anciens, quelques tables d'inverses, écrites de façon experte sur des tablettes d'argile très soignées, nous sont parvenues. De plus l'utilisation des tables métrologiques et numériques semble avoir laissé des traces dans certains textes administratifs d'Ur III sous forme de notations marginales (voir Ouyang et Proust à paraître). Cela pourrait signifier que ces listes et tables métrologiques et numériques avaient été établies oralement et transmises par la tradition orale, peut-être sous forme de comptines. Dans ce cas, leur support étant la mémoire, elles n’ont pas ou peu laissé de traces matérielles. Cette hypothèse avait été évoquée par Civil à propos des listes lexicales : "The scarcity of lexical texts during the Ur III empire is surprising. Could it be due to the strength of the oral tradition?" (Civil 1975: 127)

Les quelques exemplaires connus de tablettes scolaires mathématiques datés d'Ur III sont des œuvres d'une qualité très supérieure à celle des brouillons d'écoliers d'époque paléobabylonienne (voir Proust 2007 : 125, 242). Ce détail matériel pourrait refléter un changement dans le statut des écoles. Les écoles pourraient avoir été, sous la troisième dynastie d'Ur, des lieux de formation des officiels de haut rang, en particulier de ceux qui étaient en charge de l'administration des grands domaines royaux. A l'époque paléo-babylonienne, les écoles étaient ouvertes à des enfants ou des adolescents, peut-être nettement plus jeunes que les apprentis administrateurs d'Ur III, et semblent avoir accueilli une proportion plus large des élites en formation que dans les époques antérieures. Si de telles hypothèses étaient retenues, en particulier pour ce qui concerne une ouverture des écoles en direction d'un public plus jeune, alors certains détails des récits des textes Edubba reflèteraient bien des réalités paléobabyloniennes, et non celles de temps plus anciens. Les listes et les tables métrologiques et numériques ont pu avoir été utilisées dans l'administration dès la troisième dynastie d'Ur, ou même plus tôt. Cependant, la forme écrite cohérente, hautement structurée, et remarquablement stable que ces listes et tables présentent dans les sources paléo-babyloniennes pourrait refléter un travail de mise en forme et de théorisation par les maîtres des Edubba de cette époque, sans doute en premier lieu à Nippur. Il semble que dans les Edubba de Nippur, la main du scribe ait aussi saisi la chanson des mathématiques.

\footnotetext{
${ }^{39}$ Les arguments qui suivent ne sont pas incompatibles avec d'autres explications. Un autre changement a pu concerner la façon dont les tablettes scolaires usagées étaient éliminées ou recyclées. Les rebuts des écoles paléobabyloniennes étaient en partie réutilisés comme matériau de construction, ce qui d'une certaine manière a permis leur conservation. Les pratiques de nettoyage des tablettes usagées ont pu être différentes dans les époques antérieures, et, par exemple, conduire à leur destruction complète. Le hasard des fouilles n'est pas non plus à ignorer, comme souligné plus haut.
} 


\section{Références}

\section{Abréviations}

$\begin{array}{ll}\text { BE 20/1 } & \text { Hilprecht } 1906 \\ \text { MCT } & \text { Neugebauer et Sachs } 1945 \\ \text { TMH 8 } & \text { Proust 2008 } \\ \text { TMN } & \text { Proust 2007 }\end{array}$

\section{Bibliographie}

Attinger, Pascal. 2009. Edubbâ 1 (ETCSL 5.1.1).

Castellino, Giorgio R. 1972. Two Šulgi Hymns (B-C), Studi Semitici. Rome.

Cavigneaux, Antoine. 1983. Lexikalische Listen. Reallexikon der Assyriologie 6:609-641.

- 1999. A scholar's Library in Meturan? Dans Mesopotamian Magic, sous la direction de T. Abusch et K. van der Toorn. Groningen: Styx Publications.

Chambon, Grégory, et Eleanor Robson. 2011. Untouchable or unrepeatable? The upper end of the Old Babylonian metrological systems for capacity and area 127. Iraq $73: 127-147$.

Charpin, Dominique. 1986. Le clergé d'Ur au siècle d'Hammurabi. Genève: Droz.

- 1989. Un quartier de Nippur et le problème des écoles à l'époque paléo-babylonienne. Revue d'assyriologie et d'archéologie orientale 83:97-112.

- 1990. Un quartier de Nippur et le problème des écoles à l'époque paléo-babylonienne (suite). Revue d'assyriologie et d'archéologie orientale 84:1-16.

Civil, Miguel. 1975. Lexicography. Dans Sumerological Studies in Honor of Thorkild Jacobsen on his Seventieth Birthday, sous la direction de S. J. Lieberman. Chicago.

1985. Sur les "livres d'écoliers" à l'époque paléo-babylonienne. Dans Miscellanea Babylonica, Mélanges offerts à M. Birot, sous la direction de J.-M. Durand et J.-R. Kupper. Paris: erc.

Cooper, Jerrold. 2010. I have forgotten my burden of former days!" Forgetting the Sumerians in ancient Iraq. Journal of the American Oriental Society 130 (3):327-335.

Delnero, Paul. 2010. Sumerian Extract Tablets and Scribal Education. Journal of Cuneiform Studies 62:53-69.

2012. Memorization and the Transmission of Sumerian Literary Compositions. Journal of Near Eastern Studies 71 (2):189-208.

Friberg, Jöran. 2009. A Geometric Algorithm with Solutions to Quadratic Equations in a Sumerian Juridical Document from Ur III Umma. CDLJ 2009-3.

Gasche, Hermann. 1989. La babylonie au $17^{e}$ siècle avant notre ère: approche archéologique, problèmes et perspectives. Gand: Université de Gand.

George, Andrew R. 2005. In Search of the é.dub.ba.a: The Ancient Mesopotamian School in Literature and Reality. Dans "An Experienced Scribe Who Neglects Nothing". Ancient Near Eastern Studies in Honor of Jacob Klein, sous la direction de Y. Sefati, P. Artzi, C. Cohen, B. L. Eichler et V. A. Hurowitz. Bethesda: CDL Press.

Hilprecht, Herman Vollrat. 1906. Mathematical, Metrological and Chronological Tablets from the Temple Library of Nippur, Babylonian Expedition. Philadelphia: University of Pennsylvania. 
Klein, Jacob. 1981. The Royal Hymns of Shulgi King of Ur: Man's Quest for Immortal Fame. Transactions of the American Philosophical Society, New Series 71 (7):1-48.

Kramer, Samuel Noah. 1949. Schooldays: A Sumerian Composition Relating to the Education of a Scribe. Philadelphie: The University Museum.

Landsberger, Benno. 1956. Babylonian Scribal Craft and its Terminology. Dans Proceedings of the 23rd International Congress of Orientalists, 1954, sous la direction de D. Sinor. Cambridge: The Roayal Asiatic Society.

Lion, Brigitte, et Eleanor Robson. 2006. Quelques textes scolaires paléo-babyloniens rédigés par des femmes. Journal of Cuneiform Studies 57:37-53.

Michalowski, Piotr. 1986. Review of:Three Šulgi Hymns: Sumerian Royal Hymns Glorifying King Šulgi of Ur by Jacob Klein; The Royal Hymns of Shulgi King of Ur: Man's Quest for Immortal Fame by Jacob Klein. Journal of the American Oriental Society 106 (4):839-840.

1987. Charisma and control: on continuity and change in early Mesopotamian bureaucratic systems. Dans The organization of power, aspects of bureaucracy in the near east, sous la direction de M. Gibson et R. D. Biggs. Chicago: The Oriental Institute of the University of Chicago.

2010. Learning Music: Schooling, Apprenticeship, and Gender in Early Mesopotamia. Dans Musiker und Tradierung, sous la direction de R. Pruzsinsky et D. Shehata. Vienne : Lit.

2012. Literacy, Schooling and the Transmission of Knowledge in Early Mesopotamian Culture. Dans Theory and Practice of Knowledge Transfer. Studies in School Education in the Ancient Near East and Beyond, sous la direction de W. S. van Egmond et W. H. van Soldt. Leiden: PIHANS.

Neugebauer, Otto, et Abraham J. Sachs. 1945. Mathematical Cuneiform Texts, American Oriental Studies. New Haven: American Oriental Series \& American Schools of Oriental Research

Ouyang, Xiaoli, et Christine Proust. A paraître. Place value notations in the Ur III period: marginal numbers in administrative texts. Dans Cultures of computation and quantification, sous la direction de K. Chemla, A. Keller et C. Proust, Springer.

Proust, Christine. 2005. A propos d'un prisme du Louvre: aspects de l'enseignement des mathématiques en Mésopotamie. SCIAMVS 6:3-32.

2007. Tablettes mathématiques de Nippur. Istanbul: Institut Français d'Etudes Anatoliennes, De Boccard.

. 2008a. Les listes et tables métrologiques, entre mathématiques et lexicographie. Dans Proceedings of the 51st Rencontre Assyriologique Internationale held at the University of Chicago, July 18-22, 2005. Lexicography, Philology, and Textual Studies sous la direction de R. Biggs, J. Myers et M. Roth. Chicago: The Oriental Institute.

_ 2008b (avec la collaboration de Manfred Krebernik et Joachim Oelsner). Tablettes mathématiques de la collection Hilprecht. Leipzig: Harrassowitz.

2012. Reading colophons from Mesopotamian clay-tablets dealing with mathematics. NTM Zeitschrift für Geschichte der Wissenschaften, Technik und Medizin 20 (3):123156. 
2014. Does a Master always Write for his Students? Some Evidence from Old Babylonian Scribal Schools. Dans Scientific Sources and Teaching Contexts throughout History: Problems and Perspectives, sous la direction de A. Bernard et C. Proust. Dordrecht, Heidelberg, London, New York: Springer.

Robson, Eleanor. 2001. The Tablet House: A Scribal School in Old Babylonian Nippur. Revue d'Assyriologie 95:39-66.

- 2002. More than metrology: mathematics education in an Old Babylonian scribal school. Dans Under One Sky. Astronomy and Mathematics in the Ancient Near East, sous la direction de J. M. Steele et A. Imhausen. Münster: Ugarit-Verlag. . 2004. Mathematical cuneiform tablets in the Ashmolean Museum, Oxford. SCIAMVS 5:3-66.

- 2008. Mathematics in Ancient Iraq: A Social History. Princeton: Princeton University Press.

Tanret, Michel. 1982. Les tablettes scolaires découvertes à Tell ed-Dêr. Akkadica 27:46-49.

- 2002. Per aspera ad astra. L'apprentissage du cunéiforme à Sippar-Amnanum pendant la période paléo-babylonienne tardive, Mesopotamian History and Environment. Gand: Université de Gand.

Tinney, Steve. 1999. On the curricular setting of Sumerian literature. Iraq 61:159-172.

Veldhuis, Niek. 1997. Elementary Education at Nippur, The Lists of Trees and Wooden Objects. Ph. D. dissertation, University of Groningen, Groningen.

Wilcke, Claus. 1987. Die Inschriftenfunde der 7. und 8. Kampagnen (1983 und 1984). Dans Isin Išān Bahrīyāt III. Die Ergebnisse der Ausgrabungen 1983-1984, sous la direction de B. Hrouda. Munich : Verlag der Bayerischen Akademie der Wissenschaften. 